\title{
Analytic Approximate Solution for Falkner-Skan Equation
}

\author{
Vasile Marinca, ${ }^{1,2}$ Remus-Daniel Ene, ${ }^{3}$ and Bogdan Marinca ${ }^{4}$ \\ ${ }^{1}$ Department of Mechanics and Vibration, Politehnica University of Timişoara, 300222 Timişoara, Romania \\ ${ }^{2}$ Department of Electromechanics and Vibration, Center for Advanced and Fundamental Technical Research, \\ Romania Academy, 300223 Timișoara, Romania \\ ${ }^{3}$ Department of Mathematics, Politehnica University of Timișoara, 300006 Timișoara, Romania \\ ${ }^{4}$ Department of Applied Electronics, Politehnica University of Timişoara, 300223 Timişoara, Romania
}

Correspondence should be addressed to Remus-Daniel Ene; eneremus@gmail.com

Received 14 January 2014; Accepted 10 March 2014; Published 30 April 2014

Academic Editors: M. Han, Z. Jin, and Y. Xia

Copyright (C) 2014 Vasile Marinca et al. This is an open access article distributed under the Creative Commons Attribution License, which permits unrestricted use, distribution, and reproduction in any medium, provided the original work is properly cited.

\begin{abstract}
This paper deals with the Falkner-Skan nonlinear differential equation. An analytic approximate technique, namely, optimal homotopy asymptotic method (OHAM), is employed to propose a procedure to solve a boundary-layer problem. Our method does not depend upon small parameters and provides us with a convenient way to optimally control the convergence of the approximate solutions. The obtained results reveal that this procedure is very effective, simple, and accurate. A very good agreement was found between our approximate results and numerical solutions, which prove that OHAM is very efficient in practice, ensuring a very rapid convergence after only one iteration.
\end{abstract}

\section{Introduction}

It is known that the word "viscoelastic" means the simultaneous existence of viscous and simultaneous elastic responses of a material. Some materials having a viscoelastic behavior are relevant in many fields of study for industrial and technological applications such as polymers, plastic processing, cosmetics, geology composites, paint flow, adhesives, towers generators, accelerators, electrostatic filters, droplet filters, and the design of heat exchanges [1].

Motivated by significant applications of viscoelastic materials, a substantial amount of research works has been invested in the study of nonlinear systems. In 1931, Falkner and Skan [2] have used some approximate procedures to solve boundary-layer equations. Hartree [3] found the numerical solution using a shooting method with $F^{\prime \prime}(0)$ (see (7)) as free parameter. The boundary conditions (8) arise in the study of viscous flow past a wedge of angle $\beta \pi ; \beta>0$ corresponds to flow toward the wedge and $\beta<0$ corresponds to flow away from the wedge. The special case $\beta=0$ is called the Blasius equation where the wedge reduced to a flat plate. In $[4,5]$, it is proved that if $0 \leq \beta \leq 1$ then the FalknerSkan equation (7) with initial conditions (8) admits a unique smooth solution. For $-0.1988<\beta<0$ there exist two solutions, that is, one with $F^{\prime \prime}(0)>0$ and the other one with $F^{\prime \prime}(0)<0$. Botta et al. [6] showed that the solution of Falkner-Skan equation is unique for $\beta>1$ under the restriction $0<F^{\prime}(0)<1$. Forced convection boundarylayer flow over a wedge with uniform suction or injection is analyzed by Yih [7]. Asaithambi [8] studied the FalknerSkan equation using finite difference scheme. In [9], Zaturska and Banks presented a new solution branch in function of parameter $\beta$. This solution branch is found to end singularity at $\beta=1$; its structure is analytically investigated and the principal characteristics are described. Also the spatial stability of such solutions is commented on. The differential transformation is adopted to investigate the velocity and shear-stress fields associated with Falkner-Skan boundarylayer problem in [10]. A group of transformations is used to reduce the boundary value problem into a pair of initial value problems, which are then solved by means of the differential transformation method. The nonlinear ordinary differential equation is solved using Adomian decomposition method (ADM) by Elgazery [11] such that the condition at infinity was applied to a related Padé approximation and Laplace transformation to the obtained solution. Also ADM is used in 
[12] by Alizadeh et al. to find an analytical solution in the form of infinite power series. Magnetohydrodynamic effects on the Falkner-Skan wedge flow are studied by Abbasbandy and Hayat in [13]. The same authors used Hankel-Padé and homotopy analysis method for the derivation of the solutions [14]. From a fluid mechanical point of view, the pathophysiological situation in myocardical bridges involves fluid flow in a time dependent flow geometry caused by contracting cardiac muscles overlying an intramural segment of the coronary artery. A boundary-layer model for the calculation of the pressure drop and flow separation is presented in [15] under the assumption that the idealized flow through a constriction is given by near equilibrium velocity profiles of the FalknerSkan-Cooke family, the evolution of the boundary-layer is obtained by the simultaneous solution of the Falkner-Skan equation and the transient non-Kármán integral momentum equation.

Pirkhedri et al. [16] developed a numerical technique transforming the governing partial differential equation into a nonlinear third-order boundary value problem by similarity variables and then solved it by the rational Legendre collocation method. It used transformed Hermite-Gauss nodes as interpolation points. The steady Falkner-Skan solution for gravity-driven film flow of micropolar fluid is investigated in [17]. The ordinary differential equations are solved numerically using an implicit finite difference scheme known as the Keller-box method. In [18], Lakestani truncated the semiinfinite physical domain of the problem to a finite domain expanding the required approximate solution as the elements of Chebyshev cardinal functions. Yun proposed in [19] an iterative method for solving the Falkner-Skan equation in the form of polynomial series without requiring any differentiations or integrations of the previous iterate solutions. The author suggests a correction method which is compared with the successive differences of the iterations. In [20], Hendi and Hussain considered Falkner-Skan flow over a porous surface taking into account the case of uniform suction/blowing. Stream function formulation and suitable transformations reduce the arising problem to ordinary differential equation which has been solved by homotopy analysis method.

In science and engineering there exist a lot of nonlinear differential equations and even strongly nonlinear problems which are still very difficult to solve analytically by using traditional methods. Many methods exist for approximating the solutions of nonlinear problems, for example, the Adomian decomposition method [21], the modified Lindstedt-Poincare method [22], the parameter-expansion method [23], optimal variational method [24], optimal homotopy perturbation method [25], and so on [26].

The aim of the present paper is to propose an accurate approach to Falkner-Skan equation using an analytical technique, namely, optimal homotopy asymptotic method [2628].

The validity of our procedure, which does not imply the presence of a small parameter in the equation, is based on the construction and determination of the auxiliary functions combined with a convenient way to optimally control the convergence of the solution. The efficiency of the proposed procedure is proved while an accurate solution is explicitly analytically obtained in an iterative way after only one iteration.

\section{The Governing Equation}

The two-dimensional laminar boundary-layer equations of an incompressible fluid subject to a pressure gradient are $[2,3,9,12]$

$$
\begin{gathered}
u \frac{\partial u}{\partial x}+v \frac{\partial u}{\partial y}=-\frac{1}{\rho} p^{\prime}+v \frac{\partial^{2} u}{\partial y^{2}} \\
\frac{\partial u}{\partial x}+\frac{\partial v}{\partial y}=0
\end{gathered}
$$

where $p^{\prime}$ is the pressure gradient, $p^{\prime}=-\rho U(\partial U / \partial x), u$ is the streamwise velocity in the direction of the fluid flow, $v$ is the velocity in the direction normal to $u, v$ is the constant kinematic viscosity, and $U(x)$ is the velocity at the edge of the boundary-layer which obeys the power-law relation $U(x)=$ $a x^{m},(x>0)$, where $a$ is the mean stream velocity and $m$ is a constant. The relevant boundary conditions for fixed plate are

$$
y=0: u=0, \quad v=0 ; \quad u \longrightarrow a \text { as } y \longrightarrow \infty .
$$

A stream function $\psi(x, y)$ is introduced such that

$$
u=\frac{\partial \psi}{\partial y}, \quad v=-\frac{\partial \psi}{\partial x}
$$

Equation (2) of continuity is satisfied identically. The momentum equation (1) becomes

$$
\frac{\partial \psi}{\partial y} \frac{\partial^{2} \psi}{\partial x \partial y}-\frac{\partial \psi}{\partial x} \frac{\partial^{2} \psi}{\partial y^{2}}=U \frac{\partial U}{\partial x}+\nu \frac{\partial^{3} \psi}{\partial y^{3}} .
$$

Integrating (5) and using similarity variable yield

$$
\begin{gathered}
\psi=x^{(1+m) / 2} \sqrt{\frac{2 v a}{1+m}} F(\eta), \\
\eta=\frac{y}{x^{(1-m) / 2}} \sqrt{\frac{(1+m) a}{2 \nu}} .
\end{gathered}
$$

Substituting (6) into (5) gives the equation of FalknerSkan in the form

$$
F^{\prime \prime \prime}(\eta)+F(\eta) F^{\prime \prime}(\eta)+\beta\left(1-F^{\prime}(\eta)^{2}\right)=0
$$

with the initial and boundary conditions

$$
F(0)=0, \quad F^{\prime}(0)=0, \quad F^{\prime}(\infty)=0,
$$

where $\beta=2 m /(m+1)$ is a measure of the pressure gradient and prime denotes derivative with respect to $\eta$. 


\section{Fundamentals of the OHAM}

In what follows, we consider nonlinear differential equation

$$
L(F(\eta))+N(F(\eta))=0
$$

with boundary/initial condition

$$
B\left(F, F^{\prime}, F^{\prime \prime}, \ldots\right)=0
$$

In (9), $L$ is a linear operator and $N$ is a nonlinear operator. In (10), $B$ is a boundary operator.

According to the basic ideas of OHAM [26-28], one constructs a family of equations

$$
\begin{aligned}
(1-p) L(\mathscr{F}(\eta, p)) \\
\quad=H(\eta, p)[L(\mathscr{F}(\eta, p))+N(\mathscr{F}(\eta, p))] .
\end{aligned}
$$

The boundary condition is

$$
B\left(\mathscr{F}(\eta, p), \frac{\partial \mathscr{F}(\eta, p)}{\partial \eta}, \frac{\partial^{2} \mathscr{F}(\eta, p)}{\partial \eta^{2}}, \ldots\right)=0
$$

where $\eta \in \mathbb{R}, \mathscr{F}(\eta, p)$ is an unknown function, $p \in[0,1]$ is an embedding parameter, and $H(\eta, p)$ is an auxiliary function such that $H(\eta, 0)=0$ and $H(\eta, p) \neq 0$ for $p \neq 0$. When $p$ increases from 0 to 1 , the solution $\mathscr{F}(\eta, p)$, changes from initial approximation $F_{0}(\eta)$ to the solution $F(\eta)$. For $p=0$ and $p=1$ it holds that, respectively,

$$
\mathscr{F}(\eta, 0)=F_{0}(\eta), \quad \mathscr{F}(\eta, 1)=F(\eta) .
$$

Expanding $\mathscr{F}(\eta, p)$ in series with respect to the parameter $p$, one has

$$
\mathscr{F}(\eta, p)=F_{0}(\eta)+p F_{1}(\eta)+p^{2} F_{2}(\eta)+\cdots .
$$

The series (14) contains the auxiliary function $H(\eta, p)$ which determines their convergence region. For the auxiliary function $H(\eta, p)$ we propose that

$$
H(\eta, p)=p H_{1}\left(\eta, C_{j}\right)+p^{2} H_{2}\left(\eta, C_{j}\right)+\cdots,
$$

where $H_{i}\left(\eta, C_{j}\right)$ and $i=1,2, \ldots$ are functions of variable $\eta$ and of a number of unknown parameters $C_{j}, j=1,2, \ldots q$. In this paper we consider the $m$ th-order approximation in the form

$$
\bar{F}(\eta) \approx F_{0}(\eta)+F_{1}(\eta)+\cdots+F_{m}(\eta)
$$

Inserting (14) into (11) we obtain

$$
\begin{aligned}
L(\mathscr{F}(\eta, p))+N(\mathscr{F}(\eta, p)) & \\
= & N_{0}\left(F_{0}(\eta)\right)+p N_{1}\left(F_{0}(\eta), F_{1}(\eta)\right) \\
& +p^{2} N_{2}\left(F_{0}(\eta), F_{1}(\eta), F_{2}(\eta)\right)+\cdots,
\end{aligned}
$$

where $N_{i}\left(F_{0}, F_{1}, \ldots, F_{i}\right)$ is the coefficient of $p^{i}$ in the expansion of $L(\mathscr{F})+N(\mathscr{F})$ about the embedding parameter $p$.
Substituting (14) and (15) into (11) and equating the coefficients of like powers of $p$, we obtain the following linear equations:

$$
\begin{gathered}
L\left(F_{0}(\eta)\right)=0, \quad B\left(F_{0}(\eta), F_{0}^{\prime}(\eta), F_{0}^{\prime \prime}(\eta), \ldots\right)=0, \\
L\left(F_{i}(\eta)\right)-L\left(F_{i-1}(\eta)\right)-\sum_{j=1}^{i} H_{j} N_{i-j}\left(F_{0}, F_{1}, \ldots, F_{i-j}\right)=0 \\
B\left(F_{i}, F_{i}^{\prime}, F_{i}^{\prime \prime}, \ldots\right)=0, \quad i=1,2, \ldots, m-1, \\
L\left(F_{m}(\eta)\right)-L\left(F_{m-1}(\eta)\right)-\sum_{j=1}^{m-1} H_{j} N_{m-1-j}-H_{m} N_{0}=0 \\
B\left(F_{m}, F_{m}^{\prime}, F_{m}^{\prime \prime}, \ldots\right)=0 .
\end{gathered}
$$

At this moment, the $m$ th-order approximate solution (16) depends on the functions $H_{1}\left(\eta, C_{i}\right), H_{2}\left(\eta, C_{i}\right), \ldots, H_{m}\left(\eta, C_{i}\right)$. The parameters $C_{1}, C_{2}, \ldots, C_{q}$ which appear in the expression of $H_{i}, i=1,2, \ldots, m$ can be identified optimally via various methodologies such as the least square method, the Galerkin method, and the collocation method. The parameters $C_{1}, C_{2}, \ldots, C_{q}$ can be determined, for example, if we substitute (16) into (9), such that the residual becomes

$$
R\left(\eta, C_{i}\right)=L\left(\bar{F}\left(\eta, C_{i}\right)\right)+N\left(\bar{F}\left(\eta, C_{i}\right)\right), \quad i=1,2, \ldots, q .
$$

If $a$ and $b$ are two values from the domain of the problem and $\eta_{i} \in(a, b), i=1,2, \ldots, q$, then the residual (21) must vanish

$$
\begin{array}{r}
R\left(\eta_{1}, C_{i}\right)=R\left(\eta_{2}, C_{i}\right)=\cdots=R\left(\eta_{q}, C_{i}\right)=0, \\
i=1,2, \ldots, q
\end{array}
$$

with $q$-the number of parameters $C_{i}$ which appear in the expression of the functions $H_{j}(\eta), j=1,2, \ldots, m$.

We remark that our procedure contains the auxiliary functions $H_{1}, H_{2}, \ldots$ which provides us with a simple but rigorous way to adjust and control convergence of the solution. It must be underlined that it is very important to properly choose the functions $H_{1}, H_{2}, \ldots, H_{m}$ which appear in the $m$ th-order approximation (16). With these parameters known, the approximate solution is well determined. The parameters $C_{1}, C_{2}, \ldots$ are, namely, convergence-control parameters.

\section{Application of OHAM to Falkner-Skan Equation}

To use the basic ideas of the proposed method, we choose the linear operator

$$
L(\mathscr{F}(\eta, p))=\frac{\partial^{3} \mathscr{F}(\eta, p)}{\partial \eta^{3}}+K \frac{\partial^{2} \mathscr{F}(\eta, p)}{\partial \eta^{2}},
$$

where $K$ is the unknown parameter at this moment. 
The nonlinear operator is

$$
\begin{aligned}
N(\mathscr{F}(\eta, p))= & \mathscr{F}(\eta, p) \frac{\partial^{2} \mathscr{F}(\eta, p)}{\partial \eta^{2}}-K \frac{\partial^{2} \mathscr{F}(\eta, p)}{\partial \eta^{2}} \\
& +\beta\left[1-\left(\frac{\partial \mathscr{F}(\eta, p)}{\partial \eta}\right)^{2}\right] .
\end{aligned}
$$

The boundary conditions are

$\mathscr{F}(0, p)=0, \quad \frac{\partial \mathscr{F}(0, p)}{\partial \eta}=0, \quad \frac{\partial \mathscr{F}(\infty, p)}{\partial \eta}=1$.

Equation (18) can be written in the form

$$
\begin{gathered}
F_{0}^{\prime \prime \prime}(\eta)+K F_{0}^{\prime \prime}(\eta)=0, \\
F_{0}(0)=0, \quad F_{0}^{\prime}(0)=0, \quad F_{0}^{\prime}(\infty)=1
\end{gathered}
$$

and has the solution

$$
F_{0}(\eta)=\eta+\frac{e^{-K \eta}-1}{K}
$$

From (17), (23), and (24) one obtain the expression

$$
N_{0}(\eta)=-K F_{0}^{\prime \prime}(\eta)+F_{0}(\eta) F_{0}^{\prime \prime}(\eta)+\beta\left[1-F_{0}^{\prime}(\eta)^{2}\right] .
$$

Substituting (27) into (28), we obtain

$$
N_{0}(\eta)=(K \eta+2 \beta-1-K) e^{-K \eta}+(1-\beta) e^{-2 K \eta} .
$$

If we consider the first-order approximate solution $(m=$ 1), (16) becomes

$$
\bar{F}(\eta)=F_{0}(\eta)+F_{1}(\eta)
$$

where $F_{1}(\eta)$ is obtained from $(20)$ :

$$
F_{1}^{\prime \prime \prime}(\eta)+K F_{1}^{\prime \prime}(\eta)-\left(F_{0}^{\prime \prime \prime}(\eta)+K F_{0}^{\prime \prime}(\eta)\right)=H_{1}\left(\eta, C_{i}\right) N_{0}(\eta)
$$

Substituting (27) and (29) into (31) we obtain the equation

$$
\begin{aligned}
& F_{1}^{\prime \prime \prime}(\eta)+K F_{1}^{\prime \prime}(\eta) \\
& =H_{1}\left(\eta, C_{i}\right)\left[(K \eta+2 \beta-1-K) e^{-K \eta}+(1-\beta) e^{-2 K \eta}\right], \\
& F_{1}(0)=0, \quad F_{1}^{\prime}(0)=0, \quad F_{1}^{\prime}(\infty)=0 .
\end{aligned}
$$

There are many possibilities to choose the function $H_{1}\left(\eta, C_{i}\right)$ which appears into (32). The convergence of the solution $F_{1}(\eta)$ and consequently the convergence of the approximate solution $\bar{F}(\eta)$ given by (30) depend on the auxiliary function $H_{1}\left(\eta, C_{i}\right)$. Basically, the shape of $H_{1}\left(\eta, C_{i}\right)$ should follow the term appearing in (29) which is the product of polynomial and exponential functions. In general, we try to choose the function $H_{1}\left(\eta, C_{i}\right)$ so that the product $H_{1}\left(\eta, C_{i}\right) N_{0}(\eta)$ from (31) and $N_{0}(\eta)$ would be of the same form. In our paper, for example, we can consider only the possibilities

$$
\begin{aligned}
& H_{1}\left(\eta, C_{i}\right)=C_{1}+C_{2} \eta+C_{3} \eta^{2}+\cdots+C_{q} \eta^{q-1}, \\
& H_{1}\left(\eta, C_{i}\right)=C_{1}+C_{2} \eta+C_{3} e^{-K \eta}+C_{4} e^{-2 K \eta}, \\
& H_{1}\left(\eta, C_{i}\right)=C_{1}+C_{2} \eta+C_{3} \eta^{2}+\left(C_{4}+C_{5} \eta\right) e^{-K \eta}
\end{aligned}
$$

and so on, where $C_{1}, C_{2}, \ldots$ are unknown parameters. In the following we have four cases.

4.1. Case 1. If the auxiliary convergence-control function $H_{1}\left(\eta, C_{i}\right)$ has the form

$$
\begin{aligned}
H_{1}\left(\eta, C_{i}\right)= & C_{1}+C_{2} \eta+C_{3} \eta^{2}+C_{4} \eta^{3} \\
& +\left(C_{5}+C_{6} \eta+C_{7} \eta^{2}\right) e^{-K \eta}+C_{8} e^{-2 K \eta}
\end{aligned}
$$

then (32) can be written as

$$
\begin{aligned}
F_{1}^{\prime \prime \prime}(\eta) & +K F_{1}^{\prime \prime}(\eta) \\
= & \left\{\left(2 \beta-1-K^{2}\right) C_{1}+\left[\left(2 \beta-1-K^{2}\right) C_{2}+K C_{1}\right] \eta\right. \\
+ & {\left[\left(2 \beta-1-K^{2}\right) C_{3}+K C_{2}\right] \eta^{2} } \\
+ & {\left.\left[\left(2 \beta-1-K^{2}\right) C_{4}+K C_{3}\right] \eta^{3}+K C_{4} \eta^{4}\right\} e^{-K \eta} } \\
+ & (1-\beta) C_{1}+\left(2 \beta-1-K^{2}\right) C_{5} \\
& +\left[(1-\beta) C_{2}+K C_{5}+\left(2 \beta-1-K^{2}\right) C_{6}\right] \eta \\
& +\left[(1-\beta) C_{3}+K C_{6}+\left(2 \beta-1-K^{2}\right) C_{7}\right] \eta^{2} \\
& \left.+\left[(1-\beta) C_{4}+K C_{7}\right] \eta^{3}\right\} e^{-2 K \eta} \\
+ & (1-\beta) C_{5}+\left(2 \beta-1-K^{2}\right) C_{8} \\
+ & {\left.\left[(1-\beta) C_{6}+K C_{8}\right] \eta+(1-\beta) C_{7} \eta^{2}\right\} e^{-3 K \eta} } \\
+ & (1-\beta) C_{8} e^{-4 K \eta} .
\end{aligned}
$$

Finally, using (27) and solving (35), we determine the first-order approximate solution given by (30) in the form

$$
\begin{aligned}
\bar{F}(\eta)= & \eta-\frac{1}{K}+\frac{4 K^{2}-7 \beta-5}{4 K^{3}} C_{1}+\frac{8 K^{2}-15 \beta-17}{4 K^{4}} C_{2} \\
& +\frac{48 K^{2}-93 \beta-147}{8 K^{5}} C_{3}+\frac{96 K^{2}-189 \beta-387}{4 K^{6}} C_{4}
\end{aligned}
$$




$$
\begin{aligned}
& +\frac{9 K^{2}-14 \beta-4}{36 K^{3}} C_{5}+\frac{54 K^{2}-92 \beta-43}{216 K^{4}} C_{6} \\
& +\frac{81 K^{2}-146 \beta-97}{216 K^{5}} C_{7}+\frac{48 K^{2}-69 \beta-11}{432 K^{3}} C_{8} \\
& +\left[\frac{C_{4}}{5 K} \cdot \eta^{5}+\left(\frac{C_{3}}{4 K}+\frac{2 \beta+7-K^{2}}{4 K^{2}} C_{4}\right) \cdot \eta^{4}\right. \\
& +\left(\frac{C_{2}}{3 K}+\frac{2 \beta+5-2 K^{2}}{3 K^{2}} C_{3}\right. \\
& \left.+\frac{4 \beta+10-2 K^{2}}{K^{3}} C_{4}\right) \cdot \eta^{3} \\
& +\left(\frac{C_{1}}{2 K}+\frac{2 \beta+3-K^{2}}{2 K^{2}} C_{2}+\frac{4 \beta+7-2 K^{2}}{K^{3}} C_{3}\right. \\
& \left.+\frac{18 \beta+39-9 K^{2}}{K^{4}} C_{4}\right) \cdot \eta^{2} \\
& +\left(\frac{2 \beta+1-K^{2}}{K^{2}} C_{1}+\frac{4 \beta+4-2 K^{2}}{K^{3}} C_{2}\right. \\
& +\frac{12 \beta+18-6 K^{2}}{K^{4}} C_{3} \\
& \left.+\frac{48 \beta+96-24 K^{2}}{K^{5}} C_{4}\right) \cdot \eta \\
& +\frac{1}{K}+\frac{3 \beta+3-2 K^{2}}{2 K^{3}} C_{1}+\frac{13 \beta+19-8 K^{2}}{4 K^{4}} C_{2} \\
& +\frac{41 \beta+79-24 K^{2}}{4 K^{5}} C_{3}+\frac{339 \beta+813-192 K^{2}}{8 K^{6}} C_{4} \\
& +\frac{10 \beta+5-6 K^{2}}{12 K^{3}} C_{5}+\frac{49 \beta+41-27 K^{2}}{36 K^{4}} C_{6} \\
& +\frac{718 \beta+875-378 K^{2}}{216 K^{5}} C_{7} \\
& \left.+\frac{9 \beta+2-6 K^{2}}{36 K^{3}} C_{8}\right] \cdot e^{-K \eta} \\
& +\left[\left(\frac{\beta-1}{4 K^{3}} C_{4}-\frac{1}{4 K^{2}} C_{7}\right) \cdot \eta^{3}\right. \\
& +\left(\frac{\beta-1}{4 K^{3}} C_{3}+\frac{3 \beta-3}{2 K^{4}} C_{4}-\frac{1}{4 K^{2}} C_{6}\right. \\
& \left.+\frac{K^{2}-5-2 \beta}{4 K^{3}} C_{7}\right) \cdot \eta^{2} \\
& +\left(\frac{\beta-1}{4 K^{3}} C_{2}+\frac{\beta-1}{K^{4}} C_{3}+\frac{33 \beta-33}{8 K^{5}} C_{4}-\frac{1}{4 K^{2}} C_{5}\right. \\
& \left.+\frac{K^{2}-2 \beta-3}{4 K^{3}} C_{6}+\frac{8 K^{2}-16 \beta-25}{8 K^{4}} C_{7}\right) \cdot \eta
\end{aligned}
$$

$$
\begin{aligned}
& +\frac{\beta-1}{4 K^{3}} C_{1}+\frac{\beta-1}{2 K^{4}} C_{2}+\frac{11 \beta-11}{8 K^{5}} C_{3} \\
& +\frac{39 \beta-39}{8 K^{6}} C_{4}+\frac{K^{2}-2 \beta-1}{4 K^{3}} C_{5} \\
& \left.+\frac{4 K^{2}-8 \beta-7}{8 K^{4}} C_{6}+\frac{11 K^{2}-22 \beta-28}{8 K^{5}} C_{7}\right] \cdot e^{-2 K \eta} \\
& +\left[\frac{\beta-1}{18 K^{3}} C_{7} \eta^{2}\right. \\
& +\left(\frac{\beta-1}{18 K^{3}} C_{6}+\frac{7 \beta-7}{54 K^{4}} C_{7}-\frac{1}{18 K^{2}} C_{8}\right) \cdot \eta \\
& +\frac{\beta-1}{18 K^{3}} C_{5}+\frac{7 \beta-7}{108 K^{4}} C_{6}+\frac{11 \beta-11}{108 K^{5}} C_{7} \\
& \left.+\frac{6 K^{2}-1-12 \beta}{108 K^{3}} C_{8}\right] \cdot e^{-3 K \eta}+\frac{\beta-1}{48 K^{3}} C_{8} e^{-4 K \eta} .
\end{aligned}
$$

4.2. Case 2. The auxiliary function $H_{1}\left(\eta, C_{i}\right)$ has the form

$$
\begin{aligned}
H_{1}\left(\eta, C_{i}\right)= & C_{1}+C_{2} \eta+C_{3} \eta^{2}+C_{4} \eta^{3}+C_{5} \eta^{4} \\
& +\left(C_{6}+C_{7} \eta+C_{8} \eta^{2}\right) e^{-K \eta} .
\end{aligned}
$$

In this case, (32) becomes

$$
\begin{aligned}
F_{1}^{\prime \prime \prime}(\eta) & +K F_{1}^{\prime \prime}(\eta) \\
=\{ & \left(2 \beta-1-K^{2}\right) C_{1}+\left[\left(2 \beta-1-K^{2}\right) C_{2}+K C_{1}\right] \eta \\
+ & {\left[\left(2 \beta-1-K^{2}\right) C_{3}+K C_{2}\right] \eta^{2} } \\
+ & {\left[\left(2 \beta-1-K^{2}\right) C_{4}+K C_{3}\right] \eta^{3} } \\
+ & {\left.\left[\left(2 \beta-1-K^{2}\right) C_{5}+K C_{4}\right] \eta^{4}+K C_{5} \eta^{5}\right\} e^{-K \eta} } \\
+ & \left\{(1-\beta) C_{1}+\left(2 \beta-1-K^{2}\right) C_{6}\right. \\
& +\left[(1-\beta) C_{2}+K C_{6}+\left(2 \beta-1-K^{2}\right) C_{7}\right] \eta \\
& +\left[(1-\beta) C_{3}+K C_{7}+\left(2 \beta-1-K^{2}\right) C_{8}\right] \eta^{2} \\
& \left.+\left[(1-\beta) C_{4}+K C_{8}\right] \eta^{3}+(1-\beta) C_{5} \eta^{4}\right\} e^{-2 K \eta} \\
+ & {\left[(1-\beta) C_{6}+(1-\beta) C_{7} \eta+(1-\beta) C_{8} \eta^{2}\right] . }
\end{aligned}
$$

The first-order approximate solution (30) in this case is obtained from (38) and (27) and can be written as $\bar{F}(\eta)$

$$
\begin{gathered}
=\eta-\frac{1}{K}+\frac{4 K^{2}-7 \beta-5}{4 K^{3}} C_{1}+\frac{8 K^{2}-15 \beta-17}{4 K^{4}} C_{2} \\
+\frac{48 K^{2}-93 \beta-147}{8 K^{5}} C_{3}+\frac{96 K^{2}-189 \beta-387}{4 K^{6}} C_{4}
\end{gathered}
$$




$$
\begin{aligned}
& +\frac{960 K^{2}-1935 \beta-4785}{8 K^{7}} C_{5}+\frac{9 K^{2}-14 \beta-4}{36 K^{3}} C_{6} \\
& +\frac{54 K^{2}-92 \beta-43}{216 K^{4}} C_{7}+\frac{81 K^{2}-146 \beta-97}{216 K^{5}} C_{8} \\
& +\left[\frac{C_{5}}{6 K} \eta^{6}+\left(\frac{C_{4}}{5 K}+\frac{2 \beta+9-K^{2}}{9 K^{2}} C_{5}\right) \cdot \eta^{5}\right. \\
& +\left(\frac{C_{3}}{4 K}+\frac{2 \beta+7-K^{2}}{4 K^{2}} C_{4}+\frac{4 \beta+13-2 K^{2}}{K^{3}} C_{5}\right) \cdot \eta^{4} \\
& +\left(\frac{C_{2}}{3 K}+\frac{2 \beta+5-K^{2}}{3 K^{2}} C_{3}+\frac{4 \beta+10-2 K^{2}}{K^{3}} C_{4}\right. \\
& \left.+\frac{24 \beta+68-12 K^{2}}{K^{4}} C_{5}\right) \cdot \eta^{3} \\
& +\left(\frac{C_{1}}{2 K}+\frac{2 \beta+3-K^{2}}{2 K^{2}} C_{2}+\frac{4 \beta+7-2 K^{2}}{K^{3}} C_{3}\right. \\
& \left.+\frac{18 \beta+39-9 K^{2}}{K^{4}} C_{4}+\frac{96 \beta+252-48 K^{2}}{K^{5}} C_{5}\right) \cdot \eta^{2} \\
& +\left(\frac{2 \beta+1-K^{2}}{K^{2}} C_{1}+\frac{4 \beta+4-2 K^{2}}{K^{3}} C_{2}\right. \\
& +\frac{12 \beta+18-6 K^{2}}{K^{4}} C_{3}+\frac{48 \beta+96-24 K^{2}}{K^{5}} C_{4} \\
& \left.+\frac{240 \beta+600-120 K^{2}}{K^{6}} C_{5}\right) \cdot \eta \\
& +\frac{1}{K}+\frac{3 \beta+3-2 K^{2}}{2 K^{3}} C_{1}+\frac{13 \beta+19-8 K^{2}}{4 K^{4}} C_{2} \\
& +\frac{41 \beta+79-24 K^{2}}{4 K^{5}} C_{3}+\frac{339 \beta+813-192 K^{2}}{8 K^{6}} C_{4} \\
& +\frac{1053 \beta+2307-480 K^{2}}{4 K^{7}} C_{5}+\frac{10 \beta+5-6 K^{2}}{12 K^{3}} C_{6} \\
& +\frac{49 \beta+41-27 K^{2}}{36 K^{4}} C_{7} \\
& \left.+\frac{718 \beta+875-378 K^{2}}{216 K^{5}} C_{8}\right] \cdot e^{-K \eta} \\
& +\left[\frac{\beta-1}{4 K^{3}} C_{5} \eta^{4}+\left(\frac{\beta-1}{4 K^{3}} C_{4}-\frac{2 \beta-2}{K^{4}} C_{5}-\frac{1}{4 K^{2}} C_{8}\right) \cdot \eta^{3}\right. \\
& +\left(\frac{\beta-1}{4 K^{3}} C_{3}+\frac{3 \beta-3}{2 K^{4}} C_{4}+\frac{33 \beta-33}{4 K^{5}} C_{5}-\frac{1}{4 K^{2}} C_{7}\right. \\
& \left.+\frac{2 \beta-7-K^{2}}{4 K^{3}} C_{8}\right) \cdot \eta^{2}
\end{aligned}
$$

$$
\begin{aligned}
& +\left(\frac{\beta-1}{4 K^{3}} C_{2}+\frac{\beta-1}{K^{4}} C_{3}+\frac{33 \beta-33}{8 K^{5}} C_{4}\right. \\
& +\frac{39-39 \beta}{2 K^{6}} C_{5}-\frac{1}{4 K^{2}} C_{6}+\frac{K^{2}-2 \beta-3}{4 K^{3}} C_{7} \\
& \left.+\frac{8 K^{2}-16 \beta-25}{8 K^{4}} C_{8}\right) \cdot \eta+\frac{\beta-1}{4 K^{3}} C_{1}+\frac{\beta-1}{2 K^{4}} C_{2} \\
& +\frac{11 \beta-11}{8 K^{5}} C_{3}+\frac{39 \beta-39}{8 K^{6}} C_{4}+\frac{171 \beta-171}{8 K^{7}} C_{5} \\
& +\frac{K^{2}-2 \beta-1}{4 K^{3}} C_{6}+\frac{4 K^{2}-8 \beta-7}{8 K^{4}} C_{7} \\
& \left.+\frac{11 K^{2}-22 \beta-28}{8 K^{5}} C_{8}\right] \cdot e^{-2 K \eta} \\
& +\left[\frac{\beta-1}{18 K^{3}} C_{8} \eta^{2}+\left(\frac{\beta-1}{18 K^{3}} C_{7}+\frac{7 \beta-7}{54 K^{4}} C_{8}\right) \cdot \eta\right. \\
& \left.+\frac{\beta-1}{18 K^{3}} C_{6}+\frac{7 \beta-7}{108 K^{4}} C_{7}+\frac{11 \beta-11}{108 K^{5}} C_{8}\right] \cdot e^{-3 K \eta} .
\end{aligned}
$$

4.3. Case 3. If the auxiliary function $H_{1}\left(\eta, C_{i}\right)$ has the form

$$
\begin{aligned}
H_{1}\left(\eta, C_{i}\right)= & C_{1}+C_{2} \eta+C_{3} \eta^{2}+C_{4} \eta^{3} \\
& +\left(C_{5}+C_{6} \eta+C_{7} \eta^{2}+C_{9} \eta^{3}\right) e^{-K \eta}+C_{8} e^{-2 K \eta}
\end{aligned}
$$

then the first-order approximate solution equation (30) has the form

$$
\begin{aligned}
\overline{\bar{F}}(\eta) \bar{F}(\eta) & \begin{aligned}
&+C_{9}[- \frac{6804 K^{2}+793+908 \beta}{648 K^{6}} \\
&+ \frac{1215 K^{2}+3872+2365 \beta}{216 K^{6}} e^{-K \eta} \\
&+-\frac{1}{4 K^{2} \eta^{4}+\frac{K^{2}-7-2 \beta}{4 K^{3}} \eta^{3}} \\
&+\frac{6 K^{2}-27-12 \beta}{4 K^{4}} \eta^{2} \\
&+\frac{33 K^{2}-123-66 \beta}{8 K^{5}} \eta \\
&\left.+\frac{39 K^{2}-132-78 \beta}{8 K^{6}}\right) e^{-2 K \eta} \\
&+ \frac{\beta-1}{18 K^{3} \eta^{3}+\frac{7 \beta-7}{36 K^{4}} \eta^{2}} \\
&\left.\left.+\frac{11 \beta-11}{36 K^{5}} \eta+\frac{131 \beta-131}{648 K^{6}}\right) e^{-3 K \eta}\right]
\end{aligned}
\end{aligned}
$$

where $\bar{F}(\eta)$ is given by (36). 
4.4. Case 4. In the last case, we consider

$$
\begin{aligned}
H_{1}\left(\eta, C_{i}\right)= & C_{1}+C_{2} \eta+C_{3} \eta^{2}+C_{4} \eta^{3}+C_{5} \eta^{4} \\
& +\left(C_{6}+C_{7} \eta+C_{8} \eta^{2}+C_{9} \eta^{3}\right) e^{-K \eta}
\end{aligned}
$$

such that the first-order approximate solution equation (30) becomes

$$
\begin{aligned}
& \overline{\bar{F}}(\eta)=\bar{F}(\eta) \\
& +C_{9}\left[-\frac{6804 K^{2}+793+908 \beta}{648 K^{6}}\right. \\
& +\left(-\frac{1}{4 K^{2}} \eta^{4}+\frac{1215 K^{2}+3872+2365 \beta}{216 K^{6}} e^{-K \eta}\right. \\
& +\frac{K^{2}-7-2 \beta}{4 K^{3}} \eta^{3}+\frac{6 K^{2}-27-12 \beta}{4 K^{4}} \eta^{2} \\
& +\frac{33 K^{2}-123-66 \beta}{8 K^{5}} \eta \\
& \left.+\frac{39 K^{2}-132-78 \beta}{8 K^{6}}\right) e^{-2 K \eta} \\
& +\left(\frac{\beta-1}{18 K^{3}} \eta^{3}+\frac{7 \beta-7}{36 K^{4}} \eta^{2}+\frac{11 \beta-11}{36 K^{5}} \eta\right. \\
& \left.\left.+\frac{131 \beta-131}{648 K^{6}}\right) e^{-3 K \eta}\right] \text {, }
\end{aligned}
$$

where $\bar{F}(\eta)$ is given by (39).

\section{Numerical Examples}

In order to prove the accuracy of the obtained results, we will determine the convergence-control parameters $C_{i}$ which appear in (36), (39), (41), and (43) by means of Galerkin method. Let $R\left(\eta, C_{i}\right)$ be the residual within the approximate solution $\bar{F}(\eta)$ (or $\overline{\bar{F}}(\eta))$ given by (36), (39), (41), and (43) which satisfies (7):

$$
\begin{aligned}
R\left(\eta, C_{i}\right)= & \bar{F}^{\prime \prime \prime}\left(\eta, C_{i}\right)+\bar{F}\left(\eta, C_{i}\right) \bar{F}^{\prime \prime}\left(\eta, C_{i}\right) \\
& +\beta\left(1-\bar{F}^{\prime 2}\left(\eta, C_{i}\right)\right) .
\end{aligned}
$$

Since $R\left(\eta, C_{i}\right)$ contains the parameters $C_{i}, i=1,2, \ldots$ The parameters can be determined from the conditions

$$
\begin{aligned}
J_{i}\left(C_{j}\right) & =\int_{0}^{\infty} R\left(\eta, C_{j}\right) f_{i}(\eta) d \eta=0, \\
i & =1,2, \ldots, q, \quad j=1,2, \ldots, q,
\end{aligned}
$$

where $f_{i}$ are linear independent functions, taken as weighting functions. Equations (36) and (39) contain nine unknown parameters: $K$ and $C_{i}, i=1,2, \ldots, 8$, and therefore we consider the following nine weighting functions $(q=9)$ :

$$
\begin{aligned}
f_{1}=e^{-K \eta}, & f_{2}=\eta e^{-K \eta}, & f_{3}=\eta^{2} e^{-K \eta}, \\
f_{4}=\eta^{3} e^{-K \eta}, & f_{5}=\eta^{4} e^{-K \eta}, & f_{6}=\eta^{5} e^{-K \eta}, \\
f_{7}=\eta e^{-2 K \eta}, & f_{8}=\eta^{2} e^{-2 K \eta}, & f_{9}=\eta^{3} e^{-2 K \eta} .
\end{aligned}
$$

For (41) and (43) which contain the unknown parameters: $K, \alpha$, and $C_{i}, i=1,2, \ldots, 9$, we consider weighting functions $(q=11)$

$$
\begin{gathered}
f_{1}=e^{-K \eta}, \quad f_{2}=\eta e^{-K \eta}, \quad f_{3}=\eta^{2} e^{-K \eta}, \\
f_{4}=\eta^{3} e^{-K \eta}, \quad f_{5}=\eta^{4} e^{-K \eta}, \\
f_{6}=\eta^{4} e^{-2 K \eta}+\alpha \eta e^{-4 K \eta}, \\
f_{7}=\eta e^{-2 K \eta}, \quad f_{8}=\eta^{2} e^{-2 K \eta}, \quad f_{9}=\eta^{3} e^{-2 K \eta}, \\
f_{10}=\eta^{5} e^{-K \eta}+\eta e^{-3 K \eta}, \quad f_{11}=\eta^{3} e^{-4 K \eta}+\eta^{7} e^{-2 K \eta} .
\end{gathered}
$$

In this way, the convergence-control parameters $C_{i}, i=$ $1,2, \ldots$ are optimally determined and the first-order approximate solutions are known for different values of the known parameter $\beta$.

In what follows, we illustrate the accuracy of the OHAM comparing previously obtained approximate solutions with the numerical integration results computed by means of the shooting method combined with fourth-order Runge-Kutta method using Wolfram Mathematica 6.0 software. Also we will show that the error of the solutions decreases when the number of terms in the auxiliary convergence-control function $H_{1}$ increases. For some values of the parameter $\beta$, we will determine the approximate solutions given by (36), (39), (41), and (43) and with the unknown parameters $\alpha, K$, and $C_{i}$ obtained from the system given by (45).

Example 1. In the first case we consider that $\beta=1 / 2$.

(a) For (36) and from the system (45), following the procedure described above the convergence-control parameters are obtained

$$
\begin{gathered}
C_{1}=-0.0974633576, \quad C_{2}=0.3334342895, \\
C_{3}=-0.0846971328, \quad C_{4}=0.0054445300, \\
C_{5}=10.8640621331, \quad C_{6}=-8.9072774043, \\
C_{7}=0.7917175772, \quad C_{8}=0.4377125759, \\
K=0.9345058664
\end{gathered}
$$


TABLE 1: Comparison between OHAM results given by (49) and numerical solutions for $\beta=1 / 2$.

\begin{tabular}{lccc}
\hline$\eta$ & $F_{\text {numerical }}(\eta)$ & $\bar{F}_{\text {OHAM }}(\eta)$ from $(49)$ & Relative error $=\left|F_{\text {numerical }}(\eta)-\bar{F}_{\text {OHAM }}(\eta)\right|$ \\
\hline 0 & $-5.775724 \cdot 10^{-25}$ & $5.551115 \cdot 10^{-16}$ & $5.551115 \cdot 10^{-16}$ \\
$4 / 5$ & 0.2543480764 & 0.2543149422 & 0.0000331341 \\
$8 / 5$ & 0.8550267840 & 0.8550621314 & 0.0000353473 \\
$12 / 5$ & 1.6045273996 & 1.6043588322 & 0.0001685673 \\
$16 / 5$ & 2.3963133788 & 2.3962404320 & 0.0000729467 \\
4 & 3.1955002598 & 3.1953781616 & 0.0001220981 \\
$24 / 5$ & 3.9954529746 & 3.9949610905 & 0.0004918840 \\
$28 / 5$ & 4.7954513976 & 4.7946972370 & 0.0007541605 \\
$32 / 5$ & 5.5954513676 & 5.5946526226 & 0.0007987450 \\
$36 / 5$ & 6.3954513670 & 6.3946242000 & 0.0008271670 \\
8 & 7.1954513667 & 7.19442747097 & 0.0010238957 \\
\hline
\end{tabular}

TABLE 2: Comparison between OHAM results for velocity $\bar{F}^{\prime}(\eta)$ obtained from (49) and numerical results for $\beta=1 / 2$.

\begin{tabular}{lccc}
\hline$\eta$ & $F_{\text {numerical }}^{\prime}(\eta)$ & $\bar{F}_{\text {OHAM }}^{\prime}(\eta)$ from $(49)$ & Relative error $=\left|F_{\text {numerical }}^{\prime}(\eta)-\bar{F}_{\text {OHAM }}^{\prime}(\eta)\right|$ \\
\hline 0 & $1.852884 \cdot 10^{-21}$ & $4.440892 \cdot 10^{-16}$ & $4.440873 \cdot 10^{-16}$ \\
$4 / 5$ & 0.5833048177 & 0.5833149514 & 0.0000101337 \\
$8 / 5$ & 0.8760975697 & 0.8759651454 & 0.0001324243 \\
$12 / 5$ & 0.9760687561 & 0.9759519582 & 0.0001167979 \\
$16 / 5$ & 0.9971920750 & 0.9973768978 & 0.0001848228 \\
4 & 0.9998081986 & 0.9994693079 & 0.0003388907 \\
$24 / 5$ & 0.9999925769 & 0.9995222975 & 0.0004702794 \\
$28 / 5$ & 0.9999998398 & 0.9998331589 & 0.0001666808 \\
$32 / 5$ & 0.9999999978 & 1.0000050268 & $5.028974 \cdot 10^{-6}$ \\
$36 / 5$ & 0.9999999995 & 0.9998832648 & 0.0001167346 \\
8 & 0.9999999995 & 0.9996174949 & 0.0003825046 \\
\hline
\end{tabular}

and consequently the first-order approximate solution (36) can be written in the form

$$
\begin{aligned}
\bar{F}(\eta) & \\
=- & 0.8095502989+\eta \\
+ & (-2.2237146927+0.2852293525 \eta \\
& +0.0785389555 \eta^{2}+0.0216006979 \eta^{3} \\
& \left.+0.0115505513 \eta^{4}+0.0011652211 \eta^{5}\right) e^{-0.9345058664 \eta} \\
+ & (3.0954492754+2.0345244657 \eta \\
& \left.+0.8905286290 \eta^{2}-0.1261379297 \eta^{3}\right) e^{-1.8690117328 \eta} \\
+ & (-0.0565973734+0.2080470361 \eta \\
& \left.-0.0269476991 \eta^{2}\right) e^{-2.8035175992 \eta} \\
- & 0.0055869102 \cdot e^{-3.7380234657 \eta} .
\end{aligned}
$$

In Tables 1 and 2 we present a comparison between the first-order approximate solution given by (49) and velocity obtained from (49), respectively, with numerical results for some values of variable $\eta$ and the corresponding relative errors.

(b) From (39), obtained by means of the auxiliary convergence-control function $H_{1}$ given by (37), we obtain the following results for the parameters:

$$
\begin{gathered}
C_{1}=15.3365053132, \quad C_{2}=-20.7743165892, \\
C_{3}=9.3076203696, \quad C_{4}=-2.0941285422, \\
C_{5}=0.2277189607, \quad C_{6}=-5.2584096507, \\
C_{7}=-45.5148999446, \quad C_{8}=8.3240015527, \\
K=2.1171968259 .
\end{gathered}
$$

The first-order approximate solution (39) becomes

$$
\begin{aligned}
\bar{F}(\eta)=- & 0.8071635483+\eta \\
+ & (2.8068611339-3.4953295790 \eta \\
& +4.4303812597 \eta^{2}-2.5930631506 \eta^{3} \\
& +0.8330363534 \eta^{4}-0.1666768365 \eta^{5} \\
& \left.+0.0179261368 \eta^{6}\right) e^{-2.1171968259 \eta}
\end{aligned}
$$


TABLE 3: Comparison between OHAM results given by (51) and numerical solutions for $\beta=1 / 2$.

\begin{tabular}{lccc}
\hline$\eta$ & $F_{\text {numerical }}(\eta)$ & $\bar{F}_{\text {OHAM }}(\eta)$ from $(51)$ & Relative error $=\left|F_{\text {numerical }}(\eta)-\bar{F}_{\text {OHAM }}(\eta)\right|$ \\
\hline 0 & $-5.7757 \cdot 10^{-25}$ & $-1.3322 \cdot 10^{-15}$ & $1.3322 \cdot 10^{-15}$ \\
$4 / 5$ & 0.2543480764 & 0.2543333746 & 0.0000147017 \\
$8 / 5$ & 0.8550267840 & 0.8549561291 & 0.0000706549 \\
$12 / 5$ & 1.6045273996 & 1.6042714779 & 0.0002559216 \\
$16 / 5$ & 2.3963133788 & 2.3958902142 & 0.0004231645 \\
4 & 3.1955002598 & 3.1948843675 & 0.0006158922 \\
$24 / 5$ & 3.9954529746 & 3.9943062991 & 0.0011466754 \\
$28 / 5$ & 4.7954513976 & 4.7936944532 & 0.0017569444 \\
$32 / 5$ & 5.5954513676 & 5.5932562778 & 0.0021950898 \\
$36 / 5$ & 6.3954513670 & 6.3930163452 & 0.0024350218 \\
8 & 7.1954513667 & 7.1929060945 & 0.0025452721 \\
\hline
\end{tabular}

TABLE 4: Comparison between OHAM results for velocity $\bar{F}^{\prime}(\eta)$ obtained from (51) and numerical results for $\beta=1 / 2$.

\begin{tabular}{lccc}
\hline$\eta$ & $F_{\text {numerical }}^{\prime}(\eta)$ & $\bar{F}_{\text {OHAM }}^{\prime}(\eta)$ from $(51)$ & Relative error $=\left|F_{\text {numerical }}^{\prime}(\eta)-\bar{F}_{\text {OHAM }}^{\prime}(\eta)\right|$ \\
\hline 0 & $1.852884 \cdot 10^{-21}$ & $2.553512 \cdot 10^{-15}$ & $2.553511 \cdot 10^{-15}$ \\
$4 / 5$ & 0.5833048177 & 0.5831981849 & 0.0001066327 \\
$8 / 5$ & 0.8760975697 & 0.8760769428 & 0.0000206268 \\
$12 / 5$ & 0.9760687561 & 0.9757219753 & 0.0003467808 \\
$16 / 5$ & 0.9971920750 & 0.9970876920 & 0.0001043829 \\
4 & 0.9998081986 & 0.9993456222 & 0.0004625764 \\
$24 / 5$ & 0.9999925769 & 0.9992025737 & 0.0007900032 \\
$28 / 5$ & 0.9999998398 & 0.9993191919 & 0.0006806479 \\
$32 / 5$ & 0.9999999978 & 0.9995868200 & 0.0004131778 \\
$36 / 5$ & 0.9999999995 & 0.9997970596 & 0.0002029398 \\
8 & 0.9999999995 & 0.9999136481 & 0.0000863514 \\
\hline
\end{tabular}

$$
\begin{gathered}
+(-2.0785335331+0.0310432086 \eta \\
+0.2175675516 \eta^{2}-0.4253321356 \eta^{3} \\
\left.-0.0029993386 \eta^{4}\right) e^{-4.2343936518 \eta} \\
+(0.0788359475+0.1063683100 \eta \\
\left.-0.0243638517 \eta^{2}\right) e^{-6.3515904777 \eta}
\end{gathered}
$$

In Tables 3 and 4 we present some values of stream function (51) and velocity obtained from (51), respectively, for different values of $\eta$ and the corresponding relative errors.

(c) For (41), which depends on the auxiliary convergencecontrol function $H_{1}$ given by (40), we obtain

$$
\begin{array}{cc}
C_{1}=17.1086190380, & C_{2}=-6.1886479153, \\
C_{3}=0.7626899878, & C_{4}=-0.0317286051, \\
C_{5}=-63.8867694459, & C_{6}=0.7750251041, \\
C_{7}=-7.4945476964, & C_{8}=-9.8195426109, \\
C_{9}=-0.4164447206, & K=1.1269038309, \\
\alpha=0.8746910670 .
\end{array}
$$

Therefore, the first-order approximate solution for stream function is

$$
\begin{gathered}
\overline{\bar{F}}(\eta)=-2.4537934149+\eta \\
+(-18.9229176282+1.0563974022 \eta \\
+3.6621795605 \eta^{2}-1.0933800148 \eta^{3} \\
+0.1271627184 \eta^{4} \\
\left.+0.0056311114 \eta^{5}\right) e^{-1.1269038309 \eta} \\
+(19.9100776837+25.8158411889 \eta \\
\left.+9.3137451766 \eta^{2}+1.9358858129 \eta^{3}\right) \\
\times e^{-2.25380766196 \eta} \\
+(1.3951574877+0.7157493617 \eta \\
\left.+0.1454729886 \eta^{2}\right) e^{-3.3807114929 \eta} \\
+0.0714758716 e^{-4.5076153239 \eta}
\end{gathered}
$$

In Tables 5 and 6 we present some values of stream function (53) and velocity obtained from (53), respectively, 
TABLE 5: Comparison between OHAM results given by (53) and numerical solutions for $\beta=1 / 2$.

\begin{tabular}{lccc}
\hline$\eta$ & $F_{\text {numerical }}(\eta)$ & $\overline{\bar{F}}_{\text {OHAM }}(\eta)$ from $(53)$ & Relative error $=\left|F_{\text {numerical }}(\eta)-\overline{\bar{F}}_{\text {OHAM }}(\eta)\right|$ \\
\hline 0 & $-7.585181 \cdot 10^{-25}$ & $-2.220446 \cdot 10^{-16}$ & $2.220446 \cdot 10^{-16}$ \\
$4 / 5$ & 0.2543480764 & 0.2543410815 & $6.994904 \cdot 10^{-6}$ \\
$8 / 5$ & 0.8550267840 & 0.8550382932 & 0.0000115092 \\
$12 / 5$ & 1.6045273993 & 1.6045272608 & $1.384603 \cdot 10^{-7}$ \\
$16 / 5$ & 2.3963133781 & 2.3963351657 & 0.0000217876 \\
4 & 3.1955002585 & 3.1955812100 & 0.0000809514 \\
$24 / 5$ & 3.9954529727 & 3.9955457399 & 0.0000927671 \\
$28 / 5$ & 4.7954513949 & 4.7955738251 & 0.0001224302 \\
$32 / 5$ & 5.5954513640 & 5.5956241393 & 0.0001727753 \\
$36 / 5$ & 6.3954513623 & 6.3956765240 & 0.0002251617 \\
8 & 7.1954513606 & 7.1957387803 & 0.0002874196 \\
\hline
\end{tabular}

TABLE 6: Comparison between OHAM results for velocity $\overline{\bar{F}}^{\prime}(\eta)$ obtained from (53) and numerical results for $\beta=1 / 2$.

\begin{tabular}{lccc}
\hline$\eta$ & $F_{\text {numerical }}^{\prime}(\eta)$ & $\overline{\bar{F}}_{\text {OHAM }}^{\prime}(\eta)$ from $(53)$ & Relative error $=\left|F_{\text {numerical }}^{\prime}(\eta)-\overline{\bar{F}}_{\text {OHAM }}^{\prime}(\eta)\right|$ \\
\hline 0 & $-2.963953 \cdot 10^{-20}$ & 0 & $2.963953 \cdot 10^{-20}$ \\
$4 / 5$ & 0.5833048175 & 0.5832372756 & 0.0000675419 \\
$8 / 5$ & 0.8760975695 & 0.8761763161 & 0.0000787466 \\
$12 / 5$ & 0.9760687557 & 0.9760153463 & 0.0000534094 \\
$16 / 5$ & 0.9971920744 & 0.9972855939 & 0.0000935194 \\
4 & 0.9998081978 & 0.9998421267 & 0.0000339289 \\
$24 / 5$ & 0.9999925759 & 1.0000087749 & 0.0000161989 \\
$28 / 5$ & 0.9999998387 & 1.0000557917 & 0.0000559529 \\
$32 / 5$ & 0.9999999965 & 1.0000650747 & 0.0000650781 \\
$36 / 5$ & 0.9999999980 & 1.0000682953 & 0.0000682973 \\
8 & 0.9999999978 & 1.0000901947 & 0.0000901968 \\
\hline
\end{tabular}

for different values of variable $\eta$ and the corresponding relative errors.

Comparing the results presented in Table 1 with the results presented in Table 5 and, on the other hand, the results presented in Table 2 with the results presented in Table 6, respectively, it is clear that the analytical solutions obtained by our procedure prove to be more accurate along with an increased number of terms in the auxiliary convergencecontrol function $H_{1}$.

(d) If we consider (43) depending on the auxiliary convergence-control function $H_{1}$ given by (42) then from system (45) we obtain the following results:

$$
\begin{array}{cc}
C_{1}=28.8801485140, & C_{2}=-30.7469629234, \\
C_{3}=10.1656115824, & C_{4}=-1.3058736876, \\
C_{5}=0.0629713196, & C_{6}=-11.2295919004, \\
C_{7}=62.3525565579, & C_{8}=-18.6274524032, \\
C_{9}=13.7997786872, & K=1.9932224781, \\
\alpha=2.7977542979 .
\end{array}
$$

The first-order approximate solution for stream function (43) becomes

$$
\begin{aligned}
\overline{\bar{F}}(\eta)=- & .8042841228+\eta \\
+ & (5.055972832-7.4607384673 \eta \\
& +7.6988739549 \eta^{2}-3.9526112515 \eta^{3} \\
& +1.0002001924 \eta^{4}-0.1204170249 \eta^{5} \\
& \left.+0.0052654533 \eta^{6}\right) e^{-1.9932224781 \eta} \\
+ & -4.126293156-0.2733114331 \eta \\
& \quad-0.151609344 \eta^{2}-0.5576699112 \eta^{3} \\
& \left.\quad-0.8693555337 \eta^{4}\right) e^{-3.9864449562 \eta} \\
+ & (-0.0807202735-0.2092400883 \eta \\
& \left.\quad-0.0196584681 \eta^{2}-0.0484063456 \eta^{3}\right) \\
\times & e^{-5.9796674343 \eta} .
\end{aligned}
$$


TABLE 7: Comparison between OHAM results given by (55) and numerical solutions for $\beta=1 / 2$.

\begin{tabular}{lccc}
\hline$\eta$ & $F_{\text {numerical }}(\eta)$ & $\overline{\bar{F}}_{\text {OHAM }}(\eta)$ from $(55)$ & Relative error $=\left|F_{\text {numerical }}(\eta)-\overline{\bar{F}}_{\text {OHAM }}(\eta)\right|$ \\
\hline 0 & $-5.775724 \cdot 10^{-25}$ & $8.881784 \cdot 10^{-16}$ & $8.881784 \cdot 10^{-16}$ \\
$4 / 5$ & 0.2543480764 & 0.2543611093 & 0.0000130328 \\
$8 / 5$ & 0.8550267840 & 0.8550475945 & 0.0000208105 \\
$12 / 5$ & 1.6045273993 & 1.6045751046 & 0.0000477053 \\
$16 / 5$ & 2.3963133781 & 2.3963685295 & 0.0000551514 \\
4 & 3.1955002585 & 3.1955790615 & 0.0000788029 \\
$24 / 5$ & 3.9954529727 & 3.9955715667 & 0.0001185939 \\
$28 / 5$ & 4.7954513949 & 4.7956217908 & 0.0001703959 \\
$32 / 5$ & 5.5954513640 & 5.5956679550 & 0.0002165910 \\
$36 / 5$ & 6.3954513623 & 6.3956963294 & 0.0002449671 \\
8 & 7.1954513606 & 7.1957094147 & 0.00025805400 \\
\hline
\end{tabular}

TABLE 8: Comparison between OHAM results for velocity $\overline{\bar{F}}^{\prime}(\eta)$ obtained from (55) and numerical results for $\beta=1 / 2$.

\begin{tabular}{lccc}
\hline$\eta$ & $F_{\text {numerical }}^{\prime}(\eta)$ & $\overline{\bar{F}}_{\text {OHAM }}^{\prime}(\eta)$ from $(55)$ & Relative error $=\left|F_{\text {numerical }}^{\prime}(\eta)-\overline{\bar{F}}_{\text {OHAM }}^{\prime}(\eta)\right|$ \\
\hline 0 & $1.852884 \cdot 10^{-21}$ & $-5.329070 \cdot 10^{-15}$ & $5.329040 \cdot 10^{-15}$ \\
$4 / 5$ & 0.5833048175 & 0.5833475838 & 0.0000427662 \\
$8 / 5$ & 0.8760975695 & 0.8761071968 & $9.627358 \cdot 10^{-6}$ \\
$12 / 5$ & 0.9760687557 & 0.9760898679 & 0.0000211121 \\
$16 / 5$ & 0.99719207446 & 0.9972074735 & 0.0000153990 \\
4 & 0.9998081978 & 0.9998485848 & 0.0000403869 \\
$24 / 5$ & 0.99999257599 & 1.0000520335 & 0.0000594575 \\
$28 / 5$ & 0.99999983873 & 1.0000653592 & 0.0000655205 \\
$32 / 5$ & 0.99999999655 & 1.0000472907 & 0.0000472941 \\
$36 / 5$ & 0.99999999803 & 1.0000244710 & 0.0000244730 \\
8 & 0.9999999978 & 1.0000098270 & $9.829220 \cdot 10^{-6}$ \\
\hline
\end{tabular}

TABle 9: Comparison between the values of $\bar{F}^{\prime \prime}(0)$ obtained by means of OHAM and numerical results for $\beta=1 / 2$.

\begin{tabular}{lcccrr}
\hline Type of equation & Equation (49) & Equation (51) & Equation (53) & Equation (55) & Numerical \\
\hline $\bar{F}_{\text {OHAM }}^{\prime \prime}(0)$ & 0.92767733 & 0.92763760 & 0.92760923 & 0.92779335 & 0.92768004 \\
\hline
\end{tabular}

In Tables 7 and 8 we present a comparison between the first-order approximate solution given by (55) and velocity obtained from (55), respectively, with numerical results for some values of variable $\eta$ and the corresponding relative errors.

If we compare the results presented in Tables 3 and 7 and then the results presented in Tables 4 and 8, respectively, we can arrive at conclusion that the analytical results obtained by OHAM are more accurate along with an increased number of terms in the auxiliary convergence-control function $H_{1}$. It is important to establish the value of the shear-stress profile $F^{\prime \prime}(0)$. In Table 9 we present a comparison between the values of $\bar{F}^{\prime \prime}(0)$ obtained using OHAM from (49), (51), (53), and (55) and numerical results for $\beta=1 / 2$. Our results are in very good agreement with the numerical results.
Example 2. In this second case we suppose that $\beta=1$.

(a) For (36), from the system (45) we obtained the values of the convergence-control parameters:

$$
\begin{array}{cc}
C_{1}=5.9913279914, & C_{2}=-6.6828413743, \\
C_{3}=2.0729320860, & C_{4}=-0.1950415110, \\
C_{5}=18.0871429890, & C_{6}=-84.4868066767, \\
C_{7}=17.6775180449, & C_{8}=-0.3871636300,
\end{array}
$$$$
K=2.0064973399 \text {. }
$$ 
TABLE 10: Comparison between OHAM results given by (57) and numerical solutions for $\beta=1$.

\begin{tabular}{lccc}
\hline$\eta$ & $F_{\text {numerical }}(\eta)$ & $\bar{F}_{\text {OHAM }}(\eta)$ from $(57)$ & Relative error $=\left|F_{\text {numerical }}(\eta)-\bar{F}_{\text {OHAM }}(\eta)\right|$ \\
\hline 0 & $2.229510 \cdot 10^{-25}$ & $-1.110223 \cdot 10^{-16}$ & $1.110223 \cdot 10^{-16}$ \\
$4 / 5$ & 0.3124230332 & 0.3124218993 & $1.133942 \cdot 10^{-6}$ \\
$8 / 5$ & 0.9797795327 & 0.9797813157 & $1.783054 \cdot 10^{-6}$ \\
$12 / 5$ & 1.7552539494 & 1.7552573134 & $3.364056 \cdot 10^{-6}$ \\
$16 / 5$ & 2.5523254690 & 2.5523466484 & 0.0000211794 \\
4 & 3.3521093590 & 3.3521308223 & 0.00002146329 \\
$24 / 5$ & 4.1520998358 & 4.1521444024 & 0.0000445665 \\
$28 / 5$ & 4.9520995946 & 4.9522018532 & 0.0001022586 \\
$32 / 5$ & 5.7520995955 & 5.7522601900 & 0.0001605945 \\
$36 / 5$ & 6.5520996012 & 6.5522991212 & 0.0001995199 \\
8 & 7.3520996086 & 7.3523194836 & 0.0002198750 \\
\hline
\end{tabular}

TABLE 11: Comparison between OHAM results for velocity $\bar{F}^{\prime}(\eta)$ obtained from (57) and numerical results for $\beta=1$.

\begin{tabular}{lccc}
\hline$\eta$ & $F_{\text {numerical }}^{\prime}(\eta)$ & $\bar{F}_{\text {OHAM }}^{\prime}(\eta)$ from $(57)$ & Relative error $=\left|F_{\text {numerical }}^{\prime}(\eta)-\bar{F}_{\text {OHAM }}^{\prime}(\eta)\right|$ \\
\hline 0 & $-4.599124 \cdot 10^{-21}$ & $4.440892 \cdot 10^{-16}$ & $4.440938 \cdot 10^{-16}$ \\
$4 / 5$ & 0.6859374677 & 0.6859347711 & $2.696657 \cdot 10^{-6}$ \\
$8 / 5$ & 0.9323482529 & 0.9323468816 & $1.371273 \cdot 10^{-6}$ \\
$12 / 5$ & 0.9905493983 & 0.9905683352 & 0.00001893 \\
$16 / 5$ & 0.9991860373 & 0.9991967641 & 0.00001072 \\
4 & 0.9999584304 & 0.9999621135 & $3.683007 \cdot 10^{-6}$ \\
$24 / 5$ & 0.9999987792 & 1.0000547905 & 0.0000560112 \\
$28 / 5$ & 0.9999999836 & 1.0000795225 & 0.0000795389 \\
$32 / 5$ & 1.0000000061 & 1.0000621951 & 0.0000621890 \\
$36 / 5$ & 1.0000000081 & 1.0000356813 & 0.0000356731 \\
8 & 1.0000000101 & 1.0000168920 & 0.0000168818 \\
\hline
\end{tabular}

For these values of the parameters, from (36) we obtained the first-order approximate solution in the form

$$
\begin{aligned}
\bar{F}(\eta)= & -0.6476658539+\eta \\
+ & (1.1858804994-1.0205705493 \eta \\
& +1.1912223638 \eta^{2}-0.7433934009 \eta^{3} \\
& \left.\quad+0.1980363504 \eta^{4}-0.0194409937 \eta^{5}\right) \\
& \times e^{-2.0064973399 \eta} \\
+ & (-0.5332638964+0.2249079388 \eta \\
& \left.+0.3083821306 \eta^{2}+0.0240412688 \eta^{3}\right) \\
& \times e^{-4.0129946798 \eta} \\
+ & (-0.004950749+0.0053425041 \eta) e^{-6.0194920197 \eta}
\end{aligned}
$$

In Tables 10 and 11 we present some values of stream function given by (57) and velocity obtained from (57), respectively, for different values of variable $\eta$. Also the corresponding relative errors are given in these cases. (b) For (39), the system (45) has the solutions

$$
\begin{array}{cc}
C_{1}=25.6413506662, & C_{2}=-27.7257520445, \\
C_{3}=10.2440262047, & C_{4}=-1.6311566772, \\
C_{5}=0.1133872279, & C_{6}=-37.8620215531, \\
C_{7}=4.5810479839, & C_{8}=-9.7143709526, \\
K=2.0340821793 .
\end{array}
$$

The first-order approximate solution can be written as

$$
\begin{aligned}
\bar{F}(\eta)=- & 0.6482098275+\eta \\
+ & (1.7192094539-4.5907375859 \eta \\
& +5.3333876914 \eta^{2}-3.0101935973 \eta^{3} \\
& +0.8973516349 \eta^{4}-0.1394863598 \eta^{5} \\
& \left.+0.0092906134 \eta^{6}\right) e^{-2.0340821793 \eta}
\end{aligned}
$$


TABLE 12: Comparison between OHAM results given by (59) and numerical solutions for $\beta=1$.

\begin{tabular}{lccc}
\hline$\eta$ & $F_{\text {numerical }}(\eta)$ & $\bar{F}_{\text {OHAM }}(\eta)$ from $(59)$ & Relative error $=\left|F_{\text {numerical }}(\eta)-\bar{F}_{\text {OHAM }}(\eta)\right|$ \\
\hline 0 & $2.229510 \cdot 10^{-25}$ & $-2.220446 \cdot 10^{-16}$ & $2.220446 \cdot 10^{-16}$ \\
$4 / 5$ & 0.3124230332 & 0.3124250905 & $2.0573489 \cdot 10^{-6}$ \\
$8 / 5$ & 0.9797795327 & 0.9797816632 & $2.1304868 \cdot 10^{-6}$ \\
$12 / 5$ & 1.7552539494 & 1.7552458857 & $8.063655 \cdot 10^{-6}$ \\
$16 / 5$ & 2.5523254690 & 2.5523068656 & 0.0000186033 \\
4 & 3.3521093590 & 3.3520844463 & 0.0000249127 \\
$24 / 5$ & 4.1520998358 & 4.1520336380 & 0.0000661977 \\
$28 / 5$ & 4.9520995946 & 4.9519649131 & 0.0001346814 \\
$32 / 5$ & 5.7520995955 & 5.7518965404 & 0.0002030550 \\
$36 / 5$ & 6.5520996012 & 6.5518462101 & 0.0002533911 \\
8 & 7.3520996086 & 7.3518163433 & 0.0002832652 \\
\hline
\end{tabular}

TABLE 13: Comparison between OHAM results for velocity $\bar{F}^{\prime}(\eta)$ obtained from (59) and numerical results for $\beta=1$.

\begin{tabular}{lccc}
\hline$\eta$ & $F_{\text {numerical }}^{\prime}(\eta)$ & $\bar{F}_{\text {OHAM }}^{\prime}(\eta)$ from $(59)$ & Relative error $=\left|F_{\text {numerical }}^{\prime}(\eta)-\bar{F}_{\text {OHAM }}^{\prime}(\eta)\right|$ \\
\hline 0 & $-4.599124 \cdot 10^{-21}$ & $1.776356 \cdot 10^{-15}$ & $1.776361 \cdot 10^{-15}$ \\
$4 / 5$ & 0.6859374677 & 0.6859334525 & $4.015197 \cdot 10^{-6}$ \\
$8 / 5$ & 0.9323482529 & 0.9323592586 & 0.0000110057 \\
$12 / 5$ & 0.9905493983 & 0.9905212799 & 0.0000281183 \\
$16 / 5$ & 0.9991860373 & 0.9991867807 & $7.433778 \cdot 10^{-7}$ \\
4 & 0.9999584304 & 0.9999317943 & 0.0000266361 \\
$24 / 5$ & 0.9999987792 & 0.9999248393 & 0.0000739398 \\
$28 / 5$ & 0.9999999836 & 0.9999090229 & 0.0000909606 \\
$32 / 5$ & 1.0000000061 & 0.9999238617 & 0.0000761443 \\
$36 / 5$ & 1.0000000081 & 0.9999506141 & 0.0000493940 \\
8 & 1.0000000101 & 0.9999733687 & 0.0000266414 \\
\hline
\end{tabular}

$$
\begin{aligned}
& +(-1.0709996263+2.7307483905 \eta \\
& \left.+2.3599924746 \eta^{2}+0.5869724289 \eta^{3}\right) \\
& \times e^{-4.0681643586 \eta} .
\end{aligned}
$$

In Tables 12 and 13 we present some values of stream function (59) and velocity obtained from (59), respectively, for different values of variable $\eta$ and the corresponding relative errors.

(c) For (41) with the auxiliary convergence-control function $H_{1}$ given by $(40)$ we have

$$
\begin{gathered}
C_{1}=15.1069993472, \quad C_{2}=-6.1116892850, \\
C_{3}=0.8419581636, \quad C_{4}=-0.0391594463, \\
C_{5}=-348.1410130441, \quad C_{6}=354.6124244346, \\
C_{7}=-78.2878044901, \quad C_{8}=-3.8910409852, \\
C_{9}=-4.3179402181, \quad K=1.3940527605, \\
\alpha=1.2080121437 .
\end{gathered}
$$

The first-order approximate solution for stream function (41) becomes

$$
\begin{aligned}
\overline{\bar{F}}(\eta)=- & 0.6477776791+\eta \\
+ & (-18.001344737+2.2989361198 \eta \\
& +2.4130328127 \eta^{2}-0.8773035418 \eta^{3} \\
& \left.+0.1154431274 \eta^{4}-0.0056180723 \eta^{5}\right) \\
\times & e^{-1.3940527605 \eta} \\
+ & (18.631306321+23.515563981 \eta \\
& +12.357602161 \eta^{2}+3.3122912941 \eta^{3} \\
& \left.+0.5554669357 \eta^{4}\right) e^{-2.7881055211 \eta} \\
+ & (0.0178160913+0.1112333145 \eta) e^{-4.1821582817 \eta}
\end{aligned}
$$

In Tables 14 and 15 we present some values of stream function (61) and velocity obtained from (61), respectively, for different values of variable $\eta$ and the corresponding relative errors. 
TABLE 14: Comparison between OHAM results given by (61) and numerical solutions for $\beta=1$.

\begin{tabular}{lccc}
\hline$\eta$ & $F_{\text {numerical }}(\eta)$ & $\overline{\bar{F}}_{\text {OHAM }}(\eta)$ from $(61)$ & Relative error $=\left|F_{\text {numerical }}(\eta)-\overline{\bar{F}}_{\text {OHAM }}(\eta)\right|$ \\
\hline 0 & $4.693280 \cdot 10^{-25}$ & $6.217248 \cdot 10^{-15}$ & $6.217248 \cdot 10^{-15}$ \\
$4 / 5$ & 0.3124230332 & 0.3124256050 & $2.5717950 \cdot 10^{-6}$ \\
$8 / 5$ & 0.9797795326 & 0.9797870784 & $7.545842 \cdot 10^{-6}$ \\
$12 / 5$ & 1.7552539491 & 1.7552584440 & $4.494942 \cdot 10^{-6}$ \\
$16 / 5$ & 2.5523254682 & 2.5523369280 & 0.0000114597 \\
4 & 3.3521093576 & 3.3521193533 & $9.995766 \cdot 10^{-6}$ \\
$24 / 5$ & 4.1520998332 & 4.1521125574 & 0.0000127241 \\
$28 / 5$ & 4.9520995903 & 4.9521192859 & 0.0000196955 \\
$32 / 5$ & 5.7520995889 & 5.7521233093 & 0.0000237203 \\
$36 / 5$ & 6.5520995917 & 6.5521296538 & 0.0000300620 \\
8 & 7.3520995953 & 7.3521431339 & 0.0000435385 \\
\hline
\end{tabular}

TABLE 15: Comparison between OHAM results for velocity $\overline{\bar{F}}^{\prime}(\eta)$ obtained from (61) and numerical results for $\beta=1$.

\begin{tabular}{lccc}
\hline$\eta$ & $F_{\text {numerical }}^{\prime}(\eta)$ & $\overline{\bar{F}}_{\text {OHAM }}^{\prime}(\eta)$ from $(61)$ & Relative error $=\left|F_{\text {numerical }}^{\prime}(\eta)-\overline{\bar{F}}_{\text {OHAM }}^{\prime}(\eta)\right|$ \\
\hline 0 & $-9.390154 \cdot 10^{-21}$ & $-4.085620 \cdot 10^{-14}$ & $4.085619 \cdot 10^{-14}$ \\
$4 / 5$ & 0.6859374676 & 0.6859366849 & $7.826990 \cdot 10^{-7}$ \\
$8 / 5$ & 0.9323482527 & 0.9323490349 & $7.821535 \cdot 10^{-7}$ \\
$12 / 5$ & 0.9905493979 & 0.9905533068 & $3.908903 \cdot 10^{-6}$ \\
$16 / 5$ & 0.9991860366 & 0.9991905903 & $4.553709 \cdot 10^{-6}$ \\
4 & 0.9999584293 & 0.9999557316 & $2.697690 \cdot 10^{-6}$ \\
$24 / 5$ & 0.9999987775 & 1.0000074611 & $8.683616 \cdot 10^{-6}$ \\
$28 / 5$ & 0.9999999812 & 1.0000067856 & $6.804426 \cdot 10^{-6}$ \\
$32 / 5$ & 1.0000000029 & 1.0000048084 & $4.805536 \cdot 10^{-6}$ \\
$36 / 5$ & 1.0000000039 & 1.0000121725 & 0.0000121685 \\
8 & 1.0000000049 & 1.0000209604 & 0.0000209554 \\
\hline
\end{tabular}

(d) If we have in view (43), with the auxiliary convergence-control function $H_{1}$ given by (42), then we obtain

$$
\begin{array}{cc}
C_{1}=2.5755597425, & C_{2}=-0.5965183787, \\
C_{3}=-0.0172692621, & C_{4}=0.0136562951, \\
C_{5}=-0.0010079176, & C_{6}=-5.9530458601, \\
C_{7}=-1.9930045747, & C_{8}=-1.9931630292, \\
C_{9}=0.0743455822, & K=1.1143724942, \\
\alpha=0.2855970092
\end{array}
$$

and therefore first-order approximate solution (43) is given by

$$
\begin{aligned}
\overline{\bar{F}}(\eta)=- & 0.6475361092+\eta \\
+ & (-7.0772773941+1.5599095672 \eta \\
& +0.3833481708 \eta^{2}-0.141867195 \eta^{3} \\
& +0.0068820136 \eta^{4}+0.0015709234 \eta^{5} \\
& \left.-0.0001507451 \eta^{6}\right) e^{-1.1143724942 \eta}
\end{aligned}
$$

$$
\begin{aligned}
& +(7.7248135031+6.7700065534 \eta \\
& +2.2685016445 \eta^{2}+0.2970574871 \eta^{3} \\
& \left.+0.014966987 \eta^{4}\right) e^{-2.2287449884 \eta}
\end{aligned}
$$

In Tables 16 and 17 we present a comparison between the first-order approximate solution given by (63) and velocity obtained from (63), respectively, with numerical results and the corresponding relative errors.

If we compare the results presented in Tables 10 and 14 and then the results presented in Tables 11 and 15 we deduce that the analytical results obtained by OHAM are more accurate along with an increased number of terms in the auxiliary convergence-control function $H_{1}$. The same conclusions are deduced if we compare the results presented in Tables 12 and 16 and then the results presented in Tables 13 and 17, respectively.

In Table 18 we present a comparison between the values of $\bar{F}^{\prime \prime}(0)$ obtained using OHAM from (57), (59), (61), and (63) and numerical integration results from $\beta=1$. We can deduce that the results obtained by means of OHAM are nearly identical with those obtained through numerical integration. 
TABLE 16: Comparison between OHAM results given by (63) and numerical solutions for $\beta=1$.

\begin{tabular}{lccc}
\hline$\eta$ & $F_{\text {numerical }}(\eta)$ & $\overline{\bar{F}}_{\text {OHAM }}(\eta)$ from $(63)$ & Relative error $=\left|F_{\text {numerical }}(\eta)-\overline{\bar{F}}_{\text {OHAM }}(\eta)\right|$ \\
\hline 0 & $4.693280 \cdot 10^{-25}$ & 0 & $4.693280 \cdot 10^{-25}$ \\
$4 / 5$ & 0.3124230332 & 0.3124174580 & $5.575244 \cdot 10^{-6}$ \\
$8 / 5$ & 0.9797795326 & 0.9797740164 & $5.516204 \cdot 10^{-6}$ \\
$12 / 5$ & 1.7552539491 & 1.7552534223 & $5.267840 \cdot 10^{-7}$ \\
$16 / 5$ & 2.5523254682 & 2.5523611425 & 0.0000356742 \\
4 & 3.3521093576 & 3.3521105770 & $1.219470 \cdot 10^{-6}$ \\
$24 / 5$ & 4.1520998332 & 4.1521005519 & $7.187418 \cdot 10^{-7}$ \\
$28 / 5$ & 4.9520995903 & 4.9521462123 & 0.0000466219 \\
$32 / 5$ & 5.7520995889 & 5.7521684524 & 0.0000688634 \\
$36 / 5$ & 6.5520995917 & 6.5521510110 & 0.0000514192 \\
8 & 7.3520995953 & 7.3521263893 & 0.0000267940 \\
\hline
\end{tabular}

TABLE 17: Comparison between OHAM results for velocity $\overline{\bar{F}}^{\prime}(\eta)$ obtained from (63) and numerical results for $\beta=1$.

\begin{tabular}{lccc}
\hline$\eta$ & $F_{\text {numerical }}^{\prime}(\eta)$ & $\overline{\bar{F}}_{\text {OHAM }}^{\prime}(\eta)$ from $(63)$ & Relative error $=\left|F_{\text {numerical }}^{\prime}(\eta)-\overline{\bar{F}}_{\text {OHAM }}^{\prime}(\eta)\right|$ \\
\hline 0 & $-9.390154 \cdot 10^{-21}$ & 0 & $9.390154 \cdot 10^{-21}$ \\
$4 / 5$ & 0.6859374676 & 0.6859539897 & 0.0000165220 \\
$8 / 5$ & 0.9323482527 & 0.9323092256 & 0.0000390271 \\
$12 / 5$ & 0.9905493979 & 0.9906138617 & 0.0000644637 \\
$16 / 5$ & 0.9991860366 & 0.9991779292 & $8.107355 \cdot 10^{-6}$ \\
4 & 0.9999584293 & 0.9999145872 & 0.0000438420 \\
$24 / 5$ & 0.9999987775 & 1.0000414108 & 0.0000426333 \\
$28 / 5$ & 0.9999999812 & 1.0000539936 & 0.0000540124 \\
$32 / 5$ & 1.0000000029 & 0.9999989176 & $1.085287 \cdot 10^{-6}$ \\
$36 / 5$ & 1.0000000039 & 0.9999655183 & 0.0000344856 \\
8 & 1.0000000049 & 0.9999795865 & 0.0000204184 \\
\hline
\end{tabular}

TABLE 18: Comparison between the values of $\bar{F}^{\prime \prime}(0)$ obtained by means of OHAM and numerical results for $\beta=1$.

\begin{tabular}{lccccc}
\hline Type of equation & Equation (57) & Equation (59) & Equation (61) & Equation (63) & Numerical \\
\hline $\bar{F}_{\text {OHAM }}^{\prime \prime}(0)$ & 1.23258247 & 1.23257895 & 1.23262084 & 1.23257391 & 1.232558769 \\
\hline
\end{tabular}

Example 3. In the last case we consider $\beta=1.6$.

(a) For (36), the values of the convergence-control parameters are obtained from the system (45):

$$
\begin{gathered}
C_{1}=-4.4749746430, \quad C_{2}=2.6648959478, \\
C_{3}=-0.5277449747, \quad C_{4}=0.0344313836, \\
C_{5}=0.9084258281, \quad C_{6}=11.1816458907, \\
C_{7}=-0.5492649796, \quad C_{8}=1.5695573134, \\
K=1.7389825314 .
\end{gathered}
$$

The first-order approximate solution for stream function (36) can be written as

$$
\begin{aligned}
\bar{F}(\eta)= & -0.5440478033+\eta \\
+ & (1.6577374986-0.4175138596 \eta \\
& \quad 0.4722933401 \eta^{2}+0.2774993818 \eta^{3} \\
& \left.\quad-0.05544381588 \eta^{4}+0.0039599458 \eta^{5}\right) \\
& \times e^{-1.7389825314 \eta}
\end{aligned}
$$

$$
\begin{aligned}
+ & (-1.1629370587-1.5183251610 \eta \\
& \left.-0.7578665325 \eta^{2}-0.1287736808 \eta^{3}\right) \\
\times & e^{-3.4779650629 \eta} \\
+ & (0.0455165672+0.0373698159 \eta \\
& \left.-0.0034815689 \eta^{2}\right) e^{-5.2169475944 \eta} \\
+ & 0.0037307962 e^{-6.9559301258 \eta}
\end{aligned}
$$

In Tables 19 and 20 we present some values of the stream function given by (65) and velocity obtained from (65), respectively, for different values of variable $\eta$. Also the corresponding relative errors are given in these tables.

(b) For (39), the convergence-control parameters obtained from the system (45) have the values

$$
\begin{array}{cc}
C_{1}=12.1397319477, & C_{2}=-14.7300094090, \\
C_{3}=6.0298078722, & C_{4}=-1.0787489057,
\end{array}
$$


TABLE 19: Comparison between OHAM results given by (65) and numerical solutions for $\beta=1.6$.

\begin{tabular}{lccc}
\hline$\eta$ & $F_{\text {numerical }}(\eta)$ & $\bar{F}_{\text {OHAM }}(\eta)$ from $(65)$ & Relative error $=\left|F_{\text {numerical }}(\eta)-\bar{F}_{\text {OHAM }}(\eta)\right|$ \\
\hline 0 & $-1.692327 \cdot 10^{-25}$ & $-1.110223 \cdot 10^{-16}$ & $1.110223 \cdot 10^{-16}$ \\
$4 / 5$ & 0.3599784956 & 0.3599782796 & $2.160525 \cdot 10^{-7}$ \\
$8 / 5$ & 1.0696147641 & 1.0696156734 & $9.093019 \cdot 10^{-7}$ \\
$12 / 5$ & 1.8571623264 & 1.8571633242 & $9.977941 \cdot 10^{-7}$ \\
$16 / 5$ & 2.6560434964 & 2.6560463745 & $2.878145 \cdot 10^{-6}$ \\
4 & 3.4559804211 & 3.4559769848 & $3.436309 \cdot 10^{-6}$ \\
$24 / 5$ & 4.2559782714 & 4.2559782610 & $1.038556 \cdot 10^{-8}$ \\
$28 / 5$ & 5.0559782427 & 5.0559829283 & $4.685640 \cdot 10^{-6}$ \\
$32 / 5$ & 5.8559782660 & 5.8559798484 & $1.582358 \cdot 10^{-6}$ \\
$36 / 5$ & 6.6559783023 & 6.6559717863 & $6.516027 \cdot 10^{-6}$ \\
8 & 7.4559783545 & 7.4559638500 & 0.0000145044 \\
\hline
\end{tabular}

TABLE 20: Comparison between OHAM results for velocity $\bar{F}^{\prime}(\eta)$ obtained from (65) and numerical results for $\beta=1.6$.

\begin{tabular}{lccc}
\hline$\eta$ & $F_{\text {numerical }}^{\prime}(\eta)$ & $\bar{F}_{\text {OHAM }}^{\prime}(\eta)$ from $(65)$ & Relative error $=\left|F_{\text {numerical }}^{\prime}(\eta)-\bar{F}_{\text {OHAM }}^{\prime}(\eta)\right|$ \\
\hline 0 & $4.071713 \cdot 10^{-20}$ & $9.992007 \cdot 10^{-16}$ & $9.991600 \cdot 10^{-16}$ \\
$4 / 5$ & 0.7609225381 & 0.7609240247 & $1.486624 \cdot 10^{-6}$ \\
$8 / 5$ & 0.9619780339 & 0.9619706813 & $7.352687 \cdot 10^{-6}$ \\
$12 / 5$ & 0.9960567187 & 0.9960657372 & $9.018419 \cdot 10^{-6}$ \\
$16 / 5$ & 0.9997439284 & 0.9997365151 & $7.413383 \cdot 10^{-6}$ \\
4 & 0.9999899725 & 0.9999872482 & $2.724280 \cdot 10^{-6}$ \\
$24 / 5$ & 0.9999997837 & 1.0000081444 & $8.360731 \cdot 10^{-6}$ \\
$28 / 5$ & 1.0000000206 & 1.0000013591 & $1.338446 \cdot 10^{-6}$ \\
$32 / 5$ & 1.0000000368 & 0.9999918129 & $8.223935 \cdot 10^{-6}$ \\
$36 / 5$ & 1.0000000541 & 0.9999891905 & 0.0000108635 \\
8 & 1.0000000764 & 0.9999914106 & $8.665844 \cdot 10^{-6}$ \\
\hline
\end{tabular}

$$
\begin{gathered}
C_{5}=0.0852147302, \quad C_{6}=-14.8695758423, \\
C_{7}=-7.8344847253, \quad C_{8}=-2.4044068709, \\
K=2.0881719331 .
\end{gathered}
$$

The first-order approximate solution for stream function (39) becomes

$$
\begin{aligned}
\bar{F}(\eta)=- & 0.544241252+\eta \\
+ & (0.398631080-1.040810138 \eta \\
& +1.69949761 \eta^{2}-1.248940652 \eta^{3} \\
& +0.460677086 \eta^{4}-0.086297165 \eta^{5} \\
& \left.+0.00680138 \eta^{6}\right) e^{-2.088171933 \eta} \\
+ & (0.219770025+1.364996288 \eta \\
& +1.025546039 \eta^{2}+0.114703592 \eta^{3} \\
& \left.+0.001403806 \eta^{4}\right) e^{-4.176343866 \eta}
\end{aligned}
$$

$$
\begin{array}{r}
+(-0.0741598535-0.038516290 \eta \\
\left.-0.008802133 \eta^{2}\right) e^{-6.264515799 \eta} .
\end{array}
$$

In Tables 21 and 22 we present some values of the stream function (67) and velocity obtained from (67), respectively, and the corresponding relative errors in comparison with the numerical results.

(c) For (41), with the auxiliary convergence-control function $H_{1}$ given by (40), the system (45) has the solutions

$$
\begin{array}{cc}
C_{1}=3.7343092835, & C_{2}=-1.6919201004, \\
C_{3}=0.2585003215, & C_{4}=-0.0131947678, \\
C_{5}=26.9486507241, & C_{6}=-47.5028568351, \\
C_{7}=5.3389432018, & C_{8}=-8.7586264382, \\
C_{9}=0.2924008927, & K=1.4882721635, \\
\alpha=1974.2364175690 .
\end{array}
$$


TABLE 21: Comparison between OHAM results given by (67) and numerical solutions for $\beta=1.6$.

\begin{tabular}{lccc}
\hline$\eta$ & $F_{\text {numerical }}(\eta)$ & $\bar{F}_{\text {OHAM }}(\eta)$ from $(67)$ & Relative error $=\left|F_{\text {numerical }}(\eta)-\bar{F}_{\text {OHAM }}(\eta)\right|$ \\
\hline 0 & $-1.692327 \cdot 10^{-25}$ & $1.332267 \cdot 10^{-15}$ & $1.332267 \cdot 10^{-15}$ \\
$4 / 5$ & 0.3599784956 & 0.3599798414 & $1.345740 \cdot 10^{-6}$ \\
$8 / 5$ & 1.0696147641 & 1.0696168199 & $2.055868 \cdot 10^{-6}$ \\
$12 / 5$ & 1.8571623264 & 1.8571595718 & $2.754588 \cdot 10^{-6}$ \\
$16 / 5$ & 2.6560434964 & 2.6560360629 & $7.433444 \cdot 10^{-6}$ \\
4 & 3.4559804211 & 3.4559769853 & $3.435805 \cdot 10^{-6}$ \\
$24 / 5$ & 4.2559782714 & 4.2559486488 & 0.0000296226 \\
$28 / 5$ & 5.0559782427 & 5.0558929543 & 0.0000852884 \\
$32 / 5$ & 5.8559782660 & 5.8558366505 & 0.0001416154 \\
$36 / 5$ & 6.6559783023 & 6.6557973327 & 0.0001809696 \\
8 & 7.4559783545 & 7.4557756153 & 0.0002027391 \\
\hline
\end{tabular}

TABLE 22: Comparison between OHAM results for velocity $\bar{F}^{\prime}(\eta)$ obtained from (67) and numerical results for $\beta=1.6$.

\begin{tabular}{lccc}
\hline$\eta$ & $F_{\text {numerical }}^{\prime}(\eta)$ & $\bar{F}_{\text {OHAM }}^{\prime}(\eta)$ from $(67)$ & Relative error $=\left|F_{\text {numerical }}^{\prime}(\eta)-\bar{F}_{\text {OHAM }}^{\prime}(\eta)\right|$ \\
\hline 0 & $4.071713 \cdot 10^{-20}$ & $-3.552713 \cdot 10^{-15}$ & $3.552754 \cdot 10^{-15}$ \\
$4 / 5$ & 0.7609225381 & 0.7609190201 & $3.517984 \cdot 10^{-6}$ \\
$8 / 5$ & 0.9619780339 & 0.9619872725 & $9.238582 \cdot 10^{-6}$ \\
$12 / 5$ & 0.9960567187 & 0.9960397833 & 0.0000169354 \\
$16 / 5$ & 0.9997439284 & 0.9997513373 & $7.408827 \cdot 10^{-6}$ \\
4 & 0.9999899725 & 0.9999817723 & $8.200210 \cdot 10^{-6}$ \\
$24 / 5$ & 0.9999997837 & 0.9999433979 & 0.0000563857 \\
$28 / 5$ & 1.0000000206 & 0.9999242317 & 0.0000757889 \\
$32 / 5$ & 1.0000000368 & 0.9999386977 & 0.0000613391 \\
$36 / 5$ & 1.0000000541 & 0.9999628614 & 0.0000371926 \\
8 & 1.0000000764 & 0.9999814328 & 0.0000186436 \\
\hline
\end{tabular}

The first-order approximate solution for stream function (41) can be written as

$$
\begin{aligned}
\overline{\bar{F}}(\eta)= & -0.544001931+\eta \\
+ & (-4.354906951+1.3261535367 \eta \\
& +0.3072449473 \eta^{2}+0.1940259496 \eta^{3} \\
& \left.+0.0315309032 \eta^{4}-0.0017731659 \eta^{5}\right) \\
\times & e^{-1.4882721635 \eta} \\
+ & (4.8182102767+6.0137273891 \eta \\
& +2.4451256821 \eta^{2}+0.8109067414 \eta^{3} \\
& \left.\quad-0.0330030433 \eta^{4}\right) e^{-2.976544327 \eta}
\end{aligned}
$$

$$
\begin{aligned}
& +(0.1139109044-0.168675728 \eta \\
& \left.+0.060940104 \eta^{2}+0.0029567211 \eta^{3}\right) \\
& \times e^{-4.4648164905 \eta}-0.033212299 e^{-5.9530886541 \eta} .
\end{aligned}
$$

In Tables 23 and 24 we present some values of stream function (69) and velocity obtained from (69), respectively, for different values of variable $\eta$ and the corresponding relative errors.

(d) If we have in view (43) with the auxiliary convergencecontrol function $H_{1}$ given by (42) then the convergencecontrol parameters obtained from system (45) are

$$
\begin{array}{cc}
C_{1}=-9.9309813061, & C_{2}=6.8914081433, \\
C_{3}=-1.7496966452, & C_{4}=0.2003197512, \\
C_{5}=-0.0103677381, & C_{6}=10.1134115449, \\
C_{7}=7.0897290367, & C_{8}=1.9899138244, \\
C_{9}=0.0159776712, & K=1.5329158534, \\
\alpha=-0.9799303286 .
\end{array}
$$


TABLE 23: Comparison between OHAM results given by (69) and numerical solutions for $\beta=1.6$.

\begin{tabular}{lccc}
\hline$\eta$ & $F_{\text {numerical }}(\eta)$ & $\overline{\bar{F}}_{\text {OHAM }}(\eta)$ from $(69)$ & Relative error $=\left|F_{\text {numerical }}(\eta)-\overline{\bar{F}}_{\text {OHAM }}(\eta)\right|$ \\
\hline 0 & $-1.692327 \cdot 10^{-25}$ & $-1.693090 \cdot 10^{-15}$ & $1.693090 \cdot 10^{-15}$ \\
$4 / 5$ & 0.3599784956 & 0.3599790235 & $5.278046 \cdot 10^{-7}$ \\
$8 / 5$ & 1.0696147640 & 1.0696154385 & $6.744980 \cdot 10^{-7}$ \\
$12 / 5$ & 1.8571623261 & 1.8571642418 & $1.915749 \cdot 10^{-6}$ \\
$16 / 5$ & 2.6560434955 & 2.6560445435 & $1.048024 \cdot 10^{-6}$ \\
4 & 3.4559804190 & 3.4559816246 & $1.205614 \cdot 10^{-6}$ \\
$24 / 5$ & 4.2559782671 & 4.2559820737 & $3.806680 \cdot 10^{-6}$ \\
$28 / 5$ & 5.0559782344 & 5.0559813610 & $3.126620 \cdot 10^{-6}$ \\
$32 / 5$ & 5.8559782515 & 5.8559788900 & $6.385224 \cdot 10^{-7}$ \\
$36 / 5$ & 6.6559782783 & 6.6559788961 & $6.177966 \cdot 10^{-7}$ \\
8 & 7.4559783175 & 7.4559820586 & $3.74109314 \cdot 10^{-6}$ \\
\hline
\end{tabular}

TABLE 24: Comparison between OHAM results for velocity $\overline{\bar{F}}^{\prime}(\eta)$ obtained from (69) and numerical results for $\beta=1.6$.

\begin{tabular}{lccc}
\hline$\eta$ & $F_{\text {numerical }}^{\prime}(\eta)$ & $\overline{\bar{F}}_{\text {OHAM }}^{\prime}(\eta)$ from $(69)$ & Relative error $=\left|F_{\text {numerical }}^{\prime}(\eta)-\overline{\bar{F}}_{\text {OHAM }}^{\prime}(\eta)\right|$ \\
\hline 0 & $-2.832266 \cdot 10^{-21}$ & $2.664535 \cdot 10^{-15}$ & $2.664538 \cdot 10^{-15}$ \\
$4 / 5$ & 0.7609225380 & 0.7609232141 & $6.760967 \cdot 10^{-7}$ \\
$8 / 5$ & 0.9619780338 & 0.9619796540 & $1.620229 \cdot 10^{-6}$ \\
$12 / 5$ & 0.9960567183 & 0.9960575197 & $8.014067 \cdot 10^{-7}$ \\
$16 / 5$ & 0.9997439274 & 0.9997418375 & $2.089891 \cdot 10^{-6}$ \\
4 & 0.9999899704 & 0.9999929170 & $2.946522 \cdot 10^{-6}$ \\
$24 / 5$ & 0.9999997799 & 1.0000016372 & $1.857256 \cdot 10^{-6}$ \\
$28 / 5$ & 1.0000000145 & 0.9999969829 & $3.031551 \cdot 10^{-6}$ \\
$32 / 5$ & 1.0000000272 & 0.9999978766 & $2.150573 \cdot 10^{-6}$ \\
$36 / 5$ & 1.0000000399 & 1.0000022282 & $2.188252 \cdot 10^{-6}$ \\
8 & 1.0000000667 & 1.0000052427 & $5.176061 \cdot 10^{-6}$ \\
\hline
\end{tabular}

In this case, the first-order approximate solution for stream function (43) becomes

$$
\begin{aligned}
& \overline{\bar{F}}(\eta)=-0.5439219913+\eta \\
& +(4.650996506-2.0398248866 \eta \\
& -0.4858446135 \eta^{2}+0.550362856 \eta^{3} \\
& -0.1603616877 \eta^{4}+0.0213068753 \eta^{5} \\
& \left.-0.0011272349 \eta^{6}\right) e^{-1.5329158534 \eta} \\
& +(-4.2651113968-4.2739140037 \eta \\
& -1.660992309 \eta^{2}-0.2098183934 \eta^{3} \\
& \left.-0.0021316125 \eta^{4}\right) e^{-3.0658317069 \eta} \\
& +(0.158036881+0.0939831378 \eta \\
& \left.+0.0187520087 \eta^{2}+0.0001478554 \eta^{3}\right) \\
& \times e^{-4.5987475603 \eta} \text {. }
\end{aligned}
$$

FIGURE 1: Comparison between the approximate solutions (49), (57), and (65) and numerical results with the auxiliary function equation (34) in the cases $\beta=1 / 2,1$, and 1.6, respectively: - numerical; ...OHAM solution.

In Tables 25 and 26 we present some values of stream function given by (71) and velocity obtained from (71), respectively, for different values of variable $\eta$. Also the corresponding relative errors are given in these cases. 
TABLE 25: Comparison between OHAM results given by (71) and numerical solutions for $\beta=1.6$.

\begin{tabular}{lccc}
\hline$\eta$ & $F_{\text {numerical }}(\eta)$ & $\overline{\bar{F}}_{\text {OHAM }}(\eta)$ from $(71)$ & Relative error $=\left|F_{\text {numerical }}(\eta)-\overline{\bar{F}}_{\text {OHAM }}(\eta)\right|$ \\
\hline 0 & $-1.692327 \cdot 10^{-25}$ & $-2.331468 \cdot 10^{-15}$ & $2.331468 \cdot 10^{-15}$ \\
$4 / 5$ & 0.35997849 & 0.3599784037 & $9.195208 \cdot 10^{-8}$ \\
$8 / 5$ & 1.06961476 & 1.0696144385 & $3.254580 \cdot 10^{-7}$ \\
$12 / 5$ & 1.85716232 & 1.8571634346 & $1.108560 \cdot 10^{-6}$ \\
$16 / 5$ & 2.65604349 & 2.6560430260 & $4.694645 \cdot 10^{-7}$ \\
4 & 3.455980419 & 3.4559810382 & $6.192629 \cdot 10^{-7}$ \\
$24 / 5$ & 4.25597826 & 4.2559845299 & $6.262826 \cdot 10^{-6}$ \\
$28 / 5$ & 5.05597823 & 5.0559832266 & $4.992205 \cdot 10^{-6}$ \\
$32 / 5$ & 5.85597825 & 5.8559812915 & $3.039980 \cdot 10^{-6}$ \\
$36 / 5$ & 6.65597827 & 6.6559890626 & 0.0000107842 \\
8 & 7.4559783175 & 7.4560065554 & 0.0000282378 \\
\hline
\end{tabular}

TABLE 26: Comparison between OHAM results for velocity $\overline{\bar{F}}^{\prime}(\eta)$ obtained from (71) and numerical results for $\beta=1.6$.

\begin{tabular}{lccc}
\hline$\eta$ & $F_{\text {numerical }}^{\prime}(\eta)$ & $\overline{\bar{F}}_{\text {OHAM }}^{\prime}(\eta)$ from $(71)$ & Relative error $=\left|F_{\text {numerical }}^{\prime}(\eta)-\overline{\bar{F}}_{\text {OHAM }}^{\prime}(\eta)\right|$ \\
\hline 0 & $-2.832266 \cdot 10^{-21}$ & $3.552713 \cdot 10^{-15}$ & $3.552716 \cdot 10^{-15}$ \\
$4 / 5$ & 0.7609225380 & 0.7609232919 & $7.538631 \cdot 10^{-7}$ \\
$8 / 5$ & 0.9619780338 & 0.9619761488 & $1.885029 \cdot 10^{-6}$ \\
$12 / 5$ & 0.9960567183 & 0.9960594353 & $2.717000 \cdot 10^{-6}$ \\
$16 / 5$ & 0.9997439274 & 0.9997398342 & $4.093197 \cdot 10^{-6}$ \\
4 & 0.9999899704 & 0.9999971751 & $7.204662 \cdot 10^{-6}$ \\
$24 / 5$ & 0.9999997799 & 1.0000032843 & $3.504355 \cdot 10^{-6}$ \\
$28 / 5$ & 1.0000000145 & 0.9999951986 & $4.815848 \cdot 10^{-6}$ \\
$32 / 5$ & 1.0000000272 & 1.0000024525 & $2.425327 \cdot 10^{-6}$ \\
$36 / 5$ & 1.0000000399 & 1.0000168296 & 0.0000167896 \\
8 & 1.0000000667 & 1.0000253832 & 0.0000253165 \\
\hline
\end{tabular}

TABLE 27: Comparison between the values of $\overline{\bar{F}}^{\prime \prime}(0)$ obtained by means of OHAM and numerical results for $\beta=1.6$.

\begin{tabular}{lcccrr}
\hline Type of equation & Equation (65) & Equation (67) & Equation (69) & Equation (71) & Numerical \\
\hline$\overline{\bar{F}}_{\text {OHAM }}^{\prime \prime}(0)$ & 1.52151589 & 1.52151820 & 1.52151245 & 1.52151403 & 1.52151402 \\
\hline
\end{tabular}

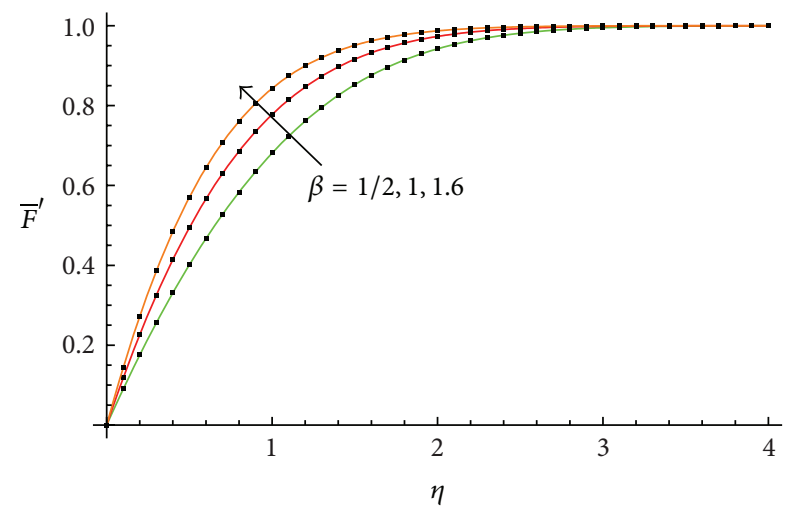

FIGURE 2: Comparison between the velocity profile obtained from (49), (57), and (65) and numerical results for $\beta=1 / 2,1$, and 1.6, respectively: - numerical; ...OHAM solution.

In Table 27 we present a comparison between the values of $\overline{\bar{F}}^{\prime \prime}(0)$ obtained through OHAM from (65), (67), (69), and (71) and numerical results for $\beta=1.6$. We can observe that the analytical solutions obtained by OHAM are very accurate, being nearly identical with the numerical results.

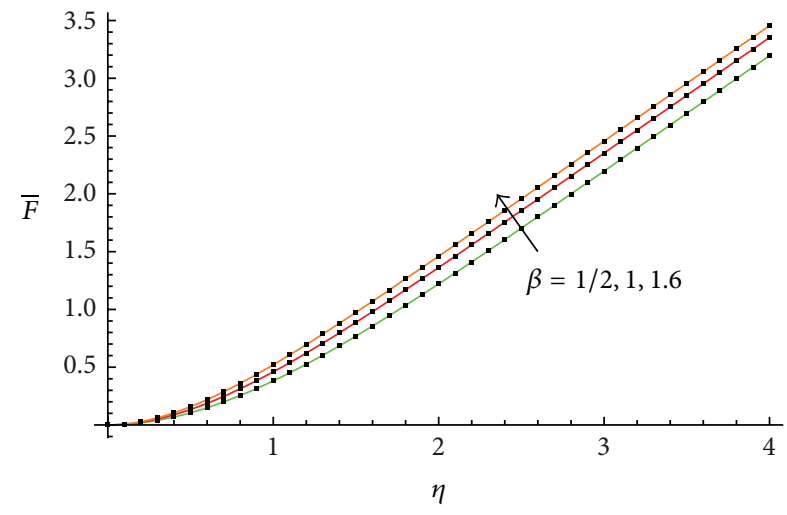

FIGURE 3: Comparison between the approximate solutions (51), (59), and (67) and numerical results with the auxiliary function equation (37) in the cases $\beta=1 / 2,1$, and 1.6, respectively: - numerical; ...OHAM solution.

The accuracy of the obtained results is verified graphically in Figures 1-8. The approximate solutions obtained by means of OHAM and with the auxiliary convergencecontrol functions $H_{1}$ given by (34), (37), (40), and (42) are compared with numerical integration results for different 


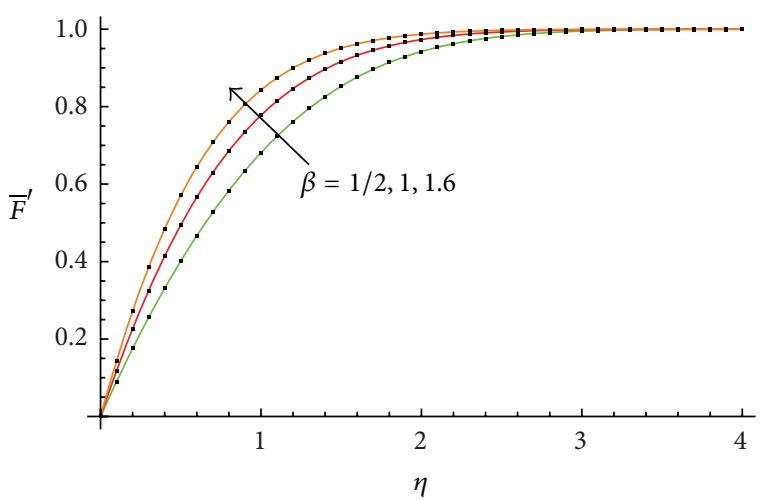

Figure 4: Comparison between the velocity profile obtained from (51), (59), and (67) and numerical results for $\beta=1 / 2,1$, and 1.6 , respectively: - numerical; ...OHAM solution.

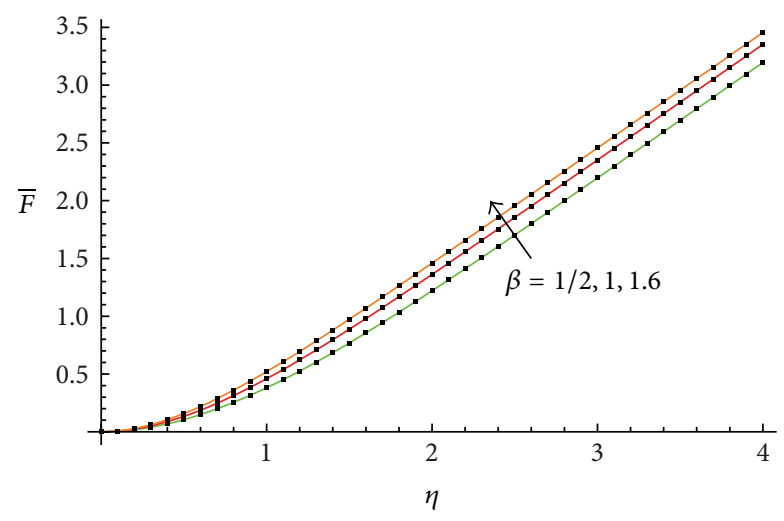

FIGURE 5: Comparison between the approximate solutions (53), (61), and (69) and numerical results with the auxiliary function equation (40) in the cases $\beta=1 / 2,1$, and 1.6, respectively: - numerical; ... OHAM solution.

values of $\beta$ in Figures $1,3,5$, and 7 , respectively. On the other hand, the velocity profile obtained from the corresponding approximate solutions is compared with numerical integration results in Figures 2, 4, 6, and 8, respectively. In all cases presented in Figures 1-8, it is shown that the firstorder approximate solutions and the velocity increase with an increase in the parameter $\beta$.

Just like the above mentioned cases, by comparing results presented in Tables 18 and 22 and then the results presented in Tables 19 and 23 we can write that the results obtained by OHAM are more accurate along with an increased number of terms in the auxiliary convergence-control function $H_{1}$. Now, comparing the results from Tables 20 and 24 and then the results from Tables 21 and 25 the conclusions are the same.

\section{Conclusions}

In the present work we proposed an optimal homotopy approach to obtain approximate analytical solutions for nonlinear differential equation of Falkner-Skan. The validity of our procedure called optimal homotopy asymptotic method (OHAM) was demonstrated on some representative

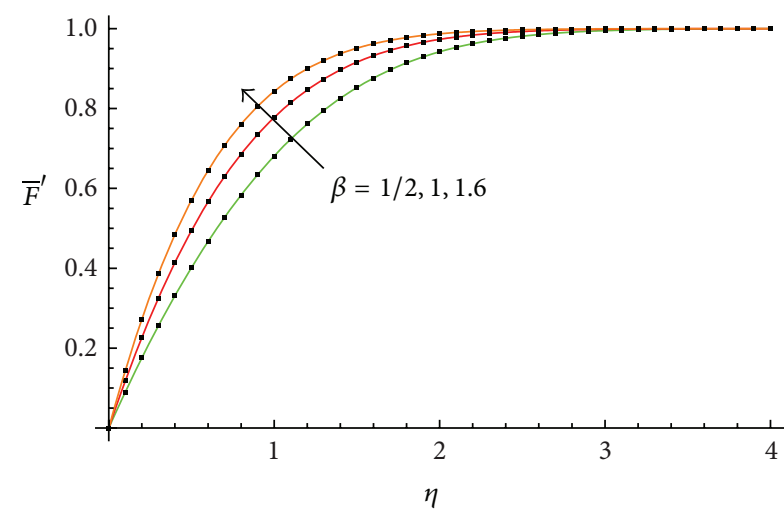

FIGURE 6: Comparison between the velocity profile obtained from (53), (61), and (69) and numerical results for $\beta=1 / 2,1$, and 1.6, respectively: - numerical; ...OHAM solution.

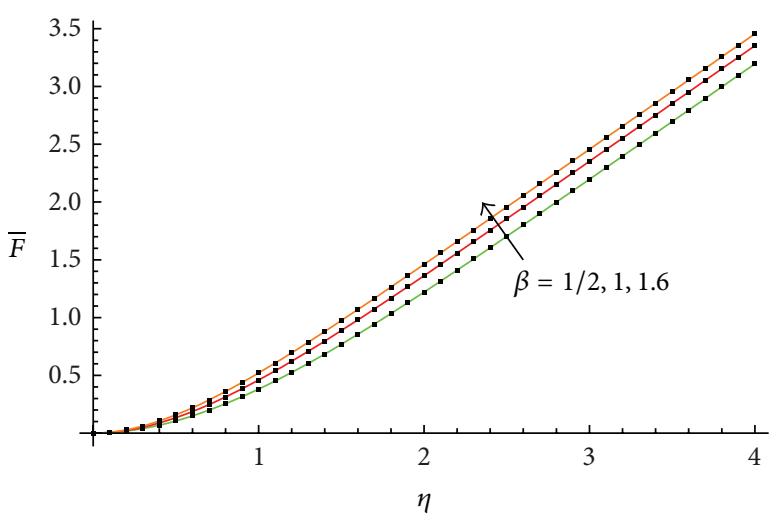

FIGURE 7: Comparison between the approximate solutions (55), (63), and (71) and numerical results with the auxiliary function equation (42) in the cases $\beta=1 / 2,1$, and 1.6, respectively: - numerical; ...OHAM solution.

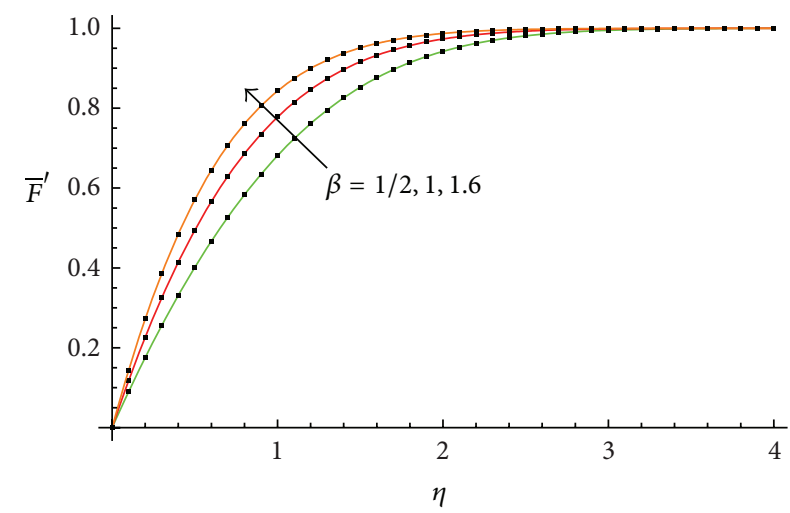

FIGURE 8: Comparison between the velocity profile obtained from (55), (63), and (71) and numerical results for $\beta=1 / 2,1$, and 1.6, respectively: - numerical; ...OHAM solution.

examples and very good agreement was found between the approximate analytic results and numerical simulation results. The proposed procedure is valid even if the nonlinear equation does not contain any small or large parameter. The basic equations governing an incompressible fluid subject to a pressure gradient are reduced to a nonlinear differential 
equation using similarity variables and are solved by means of OHAM. We examine quantitative effect of parameter $\beta$ which is a measure of the pressure gradient and the relative errors of approximate solutions in comparison with numerical results.

To solve the equation of Falkner-Skan, we used OHAM, an approach proposed by Marinca and Herişanu [26-28]. For achieving a very accurate solution, OHAM ensured a very rapid convergence after only one iteration. Our procedure is a powerful approach for solving nonlinear problems without depending on small parameters.

The cornerstone of the validity and flexibility of our method is the choice of the linear operator $L$ and the auxiliary convergence-control function $H_{1}$. The convergence of the solutions depends on these auxiliary functions and implicitly on the presence of convergence-control parameters $\alpha, K, C_{i}$, and $i=1,2, \ldots$..

Instead of an infinite series, the OHAM searches for only a few terms and does not need a recurrence formula. The parameters which appear in the composition of the auxiliary functions $H_{1}$ and in the linear operator $L$ are optimally identified via various methods by rigorously mathematical point of view. A large number of parameters in the auxiliary functions $H_{1}$ lead to a better accuracy of the results. In all cases presented in this paper, for different values of the parameter $\beta$ we obtain an excellent agreement of the firstorder approximate solutions.

Also, we obtain very good results by OHAM for different representative values of parameter $\beta$ for shear-stress profiles $\bar{F}^{\prime \prime}(0)$ of the Falkner-Skan equation in comparison with the results obtained via numerical integration. It is worth mentioning that the proposed method is straightforward and concise and can be applied to other nonlinear problems.

It is interesting to remark that a large number of parameters $C_{i}$ in the auxiliary convergence-control functions $H_{1}$ lead to a better accuracy of the results (for the stream function and velocity). On the other hand, results obtained by OHAM are more accurate along with increased values of the parameter $\beta$.

\section{Conflict of Interests}

The authors declare that there is no conflict of interests regarding the publication of this paper.

\section{References}

[1] J. de Vicente, Viscoelsticity - from Theory to Biological Applications, 2012.

[2] V. M. Falkner and S. W. Skan, "Some approximate solutions of the boundary layer equations," Philosophical Magazine, vol. 12, pp. 865-816, 1931.

[3] D. R. Hartree, "On the equation occurring in Falkner and Skan's approximate treatment of the equations of the boundary layer," Proceedings of the Cambridge Philosophical Society, vol. 33, pp. 223-239, 1937.

[4] K. Stewartson, "Further solutions of the Falkner-Skan equation," Proceedings of the Cambridge Philosophical Society, vol. 50, pp. 454-465, 1954.
[5] S. P. Hastings, "Reversed flow solutions of the Falkner-Skan equation," SIAM Journal of Applied Mathematics, vol. 22, no. 2, pp. 329-334, 1972.

[6] E. F. F. Botta, F. J. Hut, and A. E. P. Veldman, "The role of periodic solutions in the Falkner-Skan problem for $\lambda \geq 0$," Journal of Engineering Mathematics, vol. 20, no. 1, pp. 81-93, 1986.

[7] K. A. Yih, "Uniform suction/blowing effect on forced convection about a wedge: uniform heat flux," Acta Mechanica, vol. 128, no. 3-4, pp. 173-181, 1998.

[8] A. Asaithambi, "A finite difference method for the Falkner-Skan equation," Applied Mathematics and Computation, vol. 92, no. 23, pp. 135-141, 1998.

[9] M. B. Zaturska and W. N. Banks, "A new solution branch of the Falkner-Skan equation," Acta Mechanica, vol. 152, no. 1-4, pp. 197-201, 2001.

[10] B. L. Kuo, "Application of the differential transformation method to the solutions of Falkner-Skan wedge flow," Acta Mechanica, vol. 164, no. 3-4, pp. 161-174, 2003.

[11] N. S. Elgazery, "Numerical solution for the Falkner-Skan equation," Chaos, Solitons and Fractals, vol. 35, no. 4, pp. 738-746, 2008.

[12] E. Alizadeh, M. Farhadi, K. Sedighi, H. R. Ebrahimi-Kebria, and A. Ghafourian, "Solution of the Falkner-Skan equation for wedge by Adomian Decomposition Method," Communications in Nonlinear Science and Numerical Simulation, vol. 14, no. 3, pp. 724-733, 2009.

[13] S. Abbasbandy and T. Hayat, "Solution of the MHD FalknerSkan flow by Hankel-Padé method," Physics Letters A: General, Atomic and Solid State Physics, vol. 373, no. 7, pp. 731-734, 2009.

[14] S. Abbasbandy and T. Hayat, "Solution of the MHD FalknerSkan flow by homotopy analysis method," Communications in Nonlinear Science and Numerical Simulation, vol. 14, no. 9-10, pp. 3591-3598, 2009.

[15] S. Bernhard, S. Möhlenkamp, and A. Tilgner, "Transient integral boundary layer method to calculate the translesional pressure drop and the fractional flow reserve in myocardial bridges," BioMedical Engineering Online, vol. 5, article 42, 2006.

[16] A. Pirkhedri, H. H. S. Javadi, K. Parand, N. Fatahi, and S. Lotfi, "Solving MHD Falkner-Skan boundary-layer equation using collocation method based on rational Legendre function with transformed Hermite-Gauss node," World Applied Sciences Journal, vol. 13, no. 10, pp. 2224-2230, 2011.

[17] K. Ahmad, R. Nazar, and I. Pop, "Falkner-Skan solution for gravity-driven film flow of a micropolar fluid," Sains Malaysiana, vol. 40, no. 11, pp. 1291-1296, 2011.

[18] M. Lakestani, "Numerical solution for the Falkner-Skan equation using Chebyshev cardinal functions," Acta Universitas Apulensis, vol. 27, pp. 229-238, 2011.

[19] B. Y. Yun, "New approximate analytical solutions of the FalknerSkan equation," Journal of Applied Mathematics, vol. 2012, Article ID 170802, 12 pages, 2012.

[20] F. A. Hendi and M. Hussain, "Analytic solution for MHD Falkner-Skan flow over a porous surface," Journal of Applied Mathematics, vol. 2012, Article ID 123185, 9 pages, 2012.

[21] G. Adomian, "A review of the decomposition method in applied mathematics," Journal of Mathematical Analysis and Applications, vol. 135, no. 2, pp. 501-544, 1988.

[22] Y. K. Cheung, S. H. Chen, and S. L. Lau, "A modified LindstedtPoincaré method for certain strongly non-linear oscillators," International Journal of Non-Linear Mechanics, vol. 26, no. 3-4, pp. 367-378, 1991. 
[23] F. Ö. Zengin, M. O. Kaya, and S. A. Demirbağ, "Application of parameter-expansion method to nonlinear oscillators with discontinuities," International Journal of Nonlinear Sciences and Numerical Simulation, vol. 9, no. 3, pp. 267-270, 2008.

[24] V. Marinca and N. Herişanu, "Optimal variational method for truly nonlinear oscillators," Journal of Applied Mathematics, vol. 2012, Article ID 620267, 10 pages, 2012.

[25] N. Herişanu and V. Marinca, "Optimal homotopy perturbation method for non-conservative dynamical system of a rotating electrical machine," Zeitschrift für Naturforschung, vol. 670, pp. 509-516, 2012.

[26] V. Marinca and N. Herişanu, Nonlinear Dynamical Systems in Engineering-Some Approximate Approaches, Springer, Berlin, Germany, 2011.

[27] V. Marinca, N. Herişanu, C. Bota, and B. Marinca, "An optimal homotopy asymptotic method applied to the steady flow of a fourth-grade fluid past a porous plate," Applied Mathematics Letters, vol. 22, no. 2, pp. 245-251, 2009.

[28] V. Marinca and N. Herişanu, "Determination of periodic solutions for the motion of a particle on a rotating parabola by means of the optimal homotopy asymptotic method," Journal of Sound and Vibration, vol. 329, no. 9, pp. 1450-1459, 2010. 


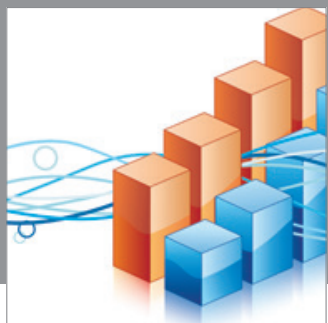

Advances in

Operations Research

mansans

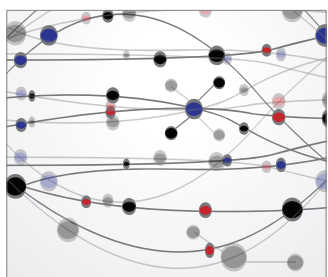

The Scientific World Journal
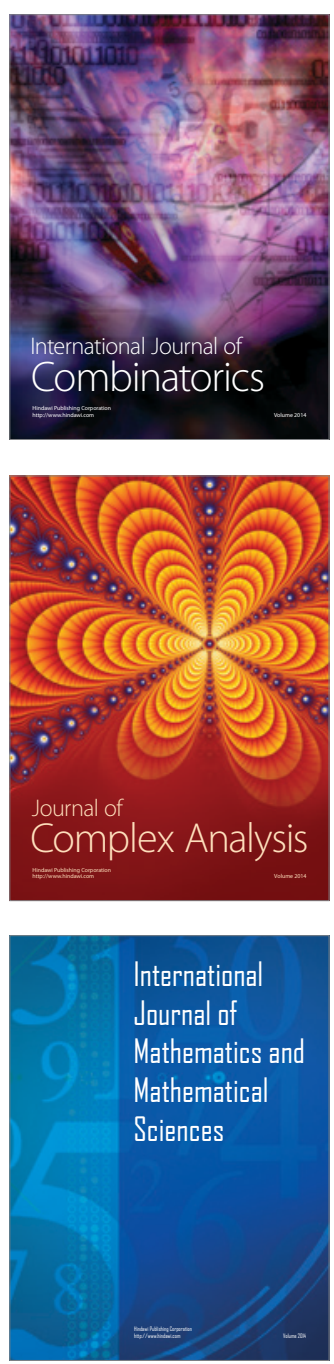
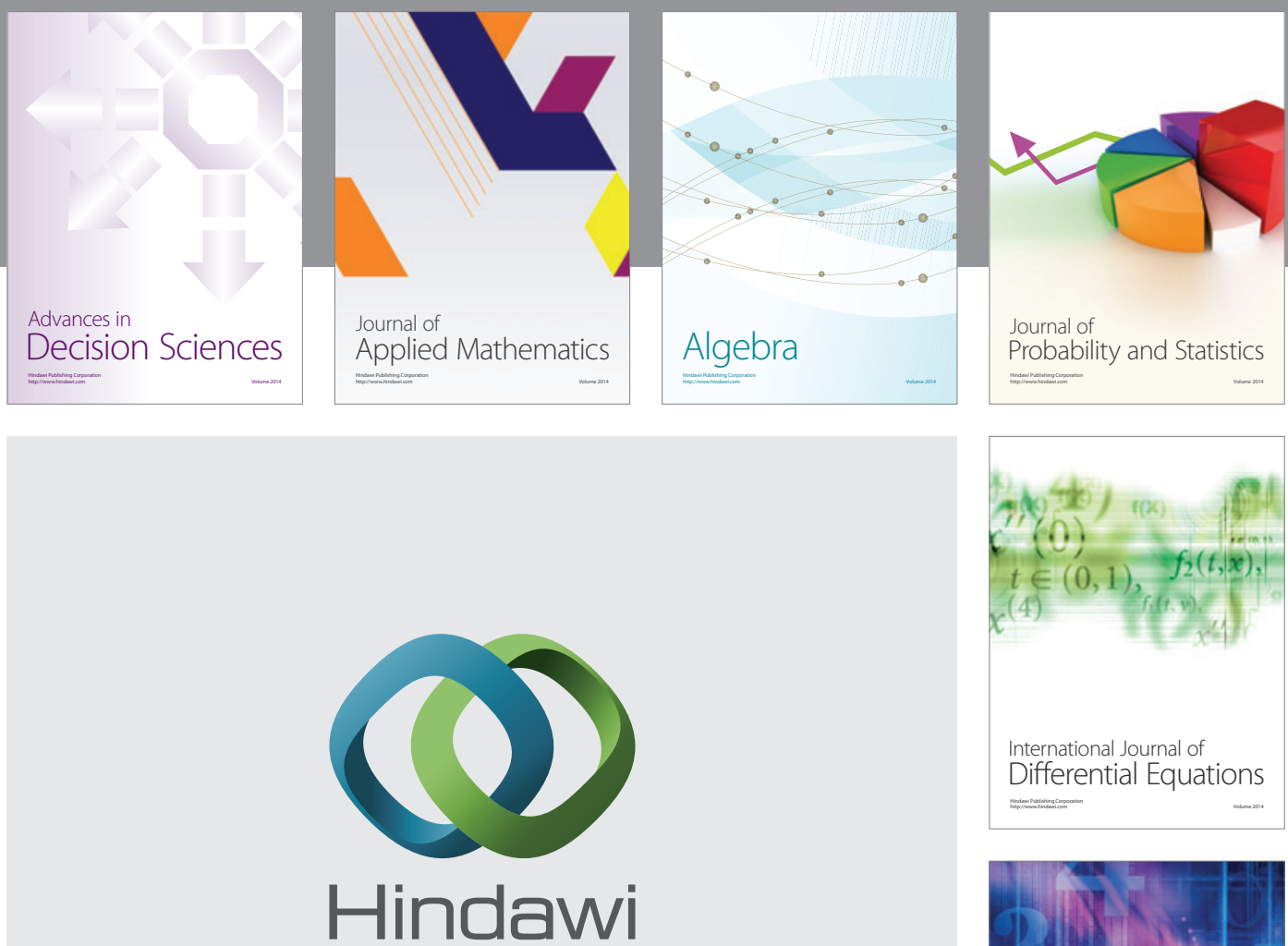

Submit your manuscripts at http://www.hindawi.com
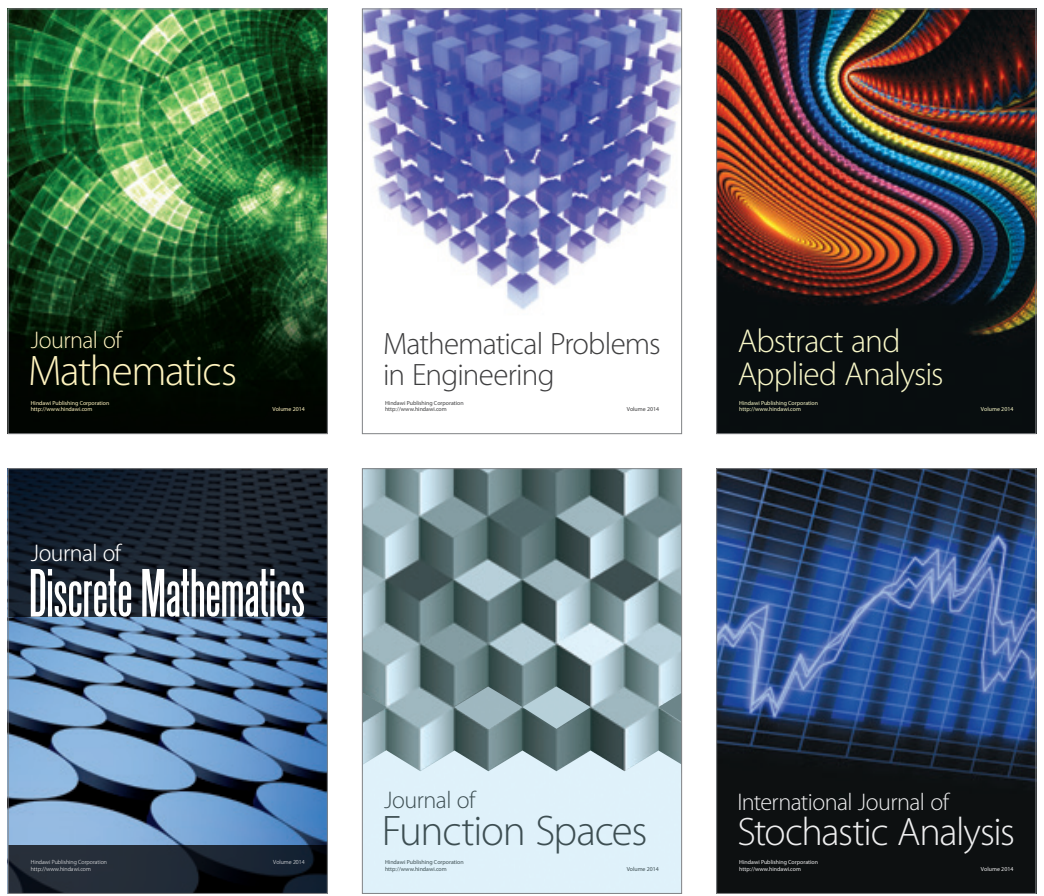

Journal of

Function Spaces

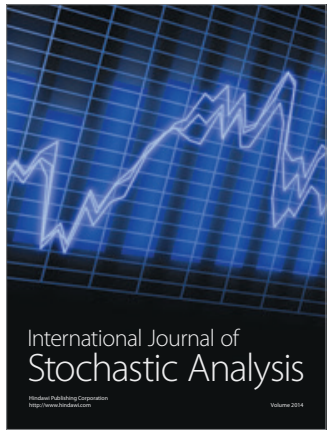

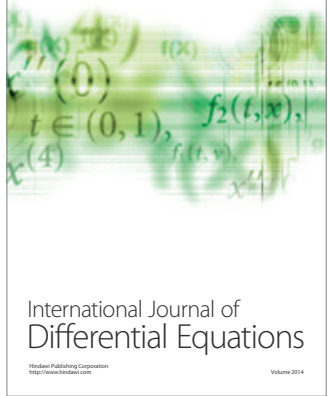
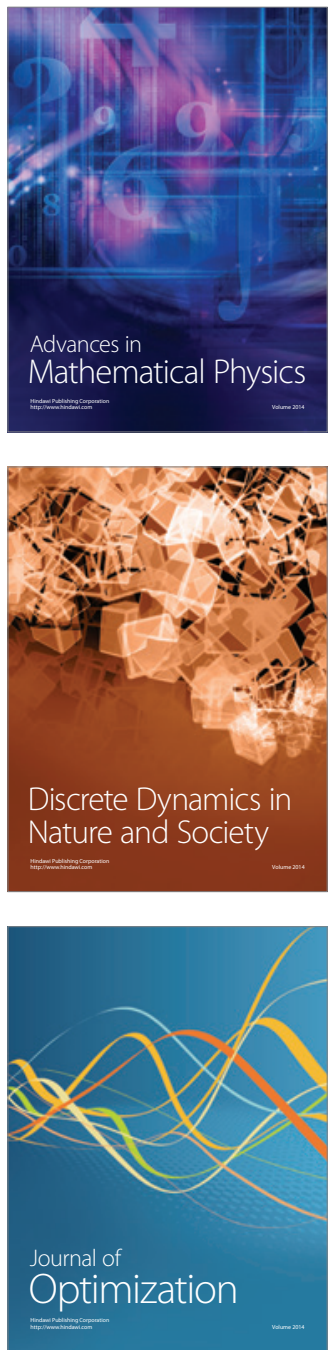